University of Nebraska - Lincoln

DigitalCommons@University of Nebraska - Lincoln

8-2005

\title{
A Link Bundled Auxiliary Graph Model for Constrained Dynamic Traffic Grooming in WDM Mesh Networks
}

Wang Yao

IEEE

Byrav Ramamurthy

University of Nebraska-Lincoln, bramamurthy2@unl.edu

Follow this and additional works at: https://digitalcommons.unl.edu/csearticles

Part of the Computer Sciences Commons

Yao, Wang and Ramamurthy, Byrav, "A Link Bundled Auxiliary Graph Model for Constrained Dynamic Traffic Grooming in WDM Mesh Networks" (2005). CSE Journal Articles. 81.

https://digitalcommons.unl.edu/csearticles/81

This Article is brought to you for free and open access by the Computer Science and Engineering, Department of at DigitalCommons@University of Nebraska - Lincoln. It has been accepted for inclusion in CSE Journal Articles by an authorized administrator of DigitalCommons@University of Nebraska - Lincoln. 


\title{
A Link Bundled Auxiliary Graph Model for Constrained Dynamic Traffic Grooming in WDM Mesh Networks
}

\author{
Wang Yao, Student Member, IEEE, and Byrav Ramamurthy, Member, IEEE
}

\begin{abstract}
This paper addresses the two-layer dynamic traffic grooming problem in wavelength-division-multiplexed (WDM) mesh optical networks subject to resource constraints and the generalized wavelength continuity (GWC) constraint. The GWC constraint is a relaxed wavelength continuity constraint which incorporates various kinds of wavelength conversion capabilities that exist in optical networks. As an improvement over the existing layered auxiliary graph (layered-AG) approach which represents each wavelength separately in the auxiliary graph, we introduce a largely simplified link bundled auxiliary graph (LBAG) model and propose the SAG-LB method to find paths and assign wavelengths for new lightpaths subject to the GWC constraint. We propose the constrained integrated grooming algorithm (CIGA) based on the LBAG model. A grooming policy influences the resource utilization by determining the weight function of the auxiliary graph. We propose the least resource path first (LR) grooming policy, which is an improvement over the existing grooming policies in the literature, by integrating the wavelength and transceiver metrics together. Simulation results show that the LBAG model achieves a comparable blocking performance with the layered-AG approach while using a significantly less amount of running time. We also present the worst case time complexity analysis of the CIGA grooming algorithm and evaluate the performance of the LR grooming policy by simulation.
\end{abstract}

Index Terms-Auxiliary graph, constrained path selection, link bundling, traffic grooming, wavelength continuity constraint, wavelength-division multiplexing (WDM).

\section{INTRODUCTION}

$\mathbf{T}$ HE EXPLOSIVELY growing Internet demands a large volume of network bandwidth. Wavelength-divisionmultiplexing (WDM) technology is a cost-effective approach to meet this need. WDM technology provides huge bandwidth in a fiber by allowing simultaneous transmission of traffic on multiple wavelengths in the fiber. While the transmission capacity of a single wavelength reaches $10 \mathrm{~Gb} / \mathrm{s}(\mathrm{OC}-192)$ or even higher, the capacity requirement of a connection (or circuit) request may be far less than that, possibly as low as $155 \mathrm{Mb} / \mathrm{s}$ (OC-3) or $622 \mathrm{Mb} / \mathrm{s}(\mathrm{OC}-12)$. Thus, an essential functionality of a WDM network, referred to as traffic grooming [1]-[5], is to aggregate low speed traffic connections onto high-speed

Manuscript received May 27, 2004; revised February 10, 2005. This work was supported in part by the National Science Foundation (NSF) under Grant ANI-0074121 and Grant EPS-0091900. This paper was presented in part at the IEEE HPSR 2004, Phoenix, AZ, April 2004.

The authors are with the Department of Computer Science and Engineering, University of Nebraska-Lincoln, Lincoln NE 68588 USA (e-mail: wyao@ cse.unl.edu; byrav@cse.unl.edu).

Digital Object Identifier 10.1109/JSAC.2005.851792

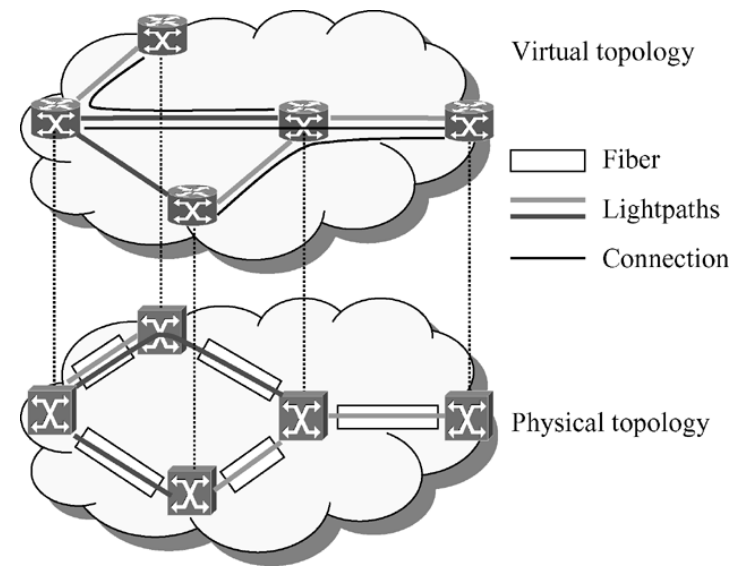

Fig. 1. Illustration of two-layer traffic grooming.

wavelength channels in a resource-efficient way, that is, to minimize the resources consumed when the connection requests to be satisfied are given or to maximize the network throughput when the resources are given.

In a wavelength-routed network, optical cross-connects (OXCs) are connected by fiber links. All the fiber links form the physical topology of the optical network. A wavelength path is referred to as a lightpath, which may span several physical links and uses one wavelength on each link along its path. At the source node of a lightpath, a transmitter is used to generate an optical signal from an electronic signal. At the destination node of a lightpath, a receiver is used to convert the optical signal into an electronic signal. All the lightpaths established over the physical topology form the virtual topology. The traffic connections are routed over the virtual topology. Therefore, the traffic grooming problem in wavelength-routed networks is generally a two-layer routing problem with connections routed over lightpaths in the virtual topology layer and lightpaths routed over physical links in the physical topology layer, as illustrated in Fig. 1.

For the dynamic traffic grooming problem at the network operation stage, connection requests arrive and depart dynamically. As resources have already been deployed in the network and will remain unchanged for some time, the objective of a dynamic traffic grooming algorithm is to maximize network throughput, or minimize the blocking probability of connection requests. To achieve this objective, the grooming algorithm must provision resource-efficient routes for both lightpaths and connections. 
Depending on the wavelength conversion [6] capability of the OXCs, a lightpath may or may not be required to use the same wavelength along its path. For example, in an all-optical wavelength-routed network without wavelength conversion capability, the wavelength continuity constraint must be enforced. Alternatively, in a wavelength-routed network deployed with $\mathrm{O} / \mathrm{E} / \mathrm{O}$ OXCs, where full wavelength conversion capability is available, a lightpath may use any wavelength available on the links along its path. Because wavelength conversion capability relaxes the wavelength continuity constraint, more lightpaths may be established in a network with wavelength conversion capability than in a network without wavelength conversion capability. However, in practice, limited-range wavelength conversion [7] may be utilized instead of full wavelength conversion, because the latter is technically more difficult to achieve in the optical domain than the former one. Because wavelength converters are still relatively expensive devices, it might be more practical to deploy wavelength conversion capability in a small fraction of the network nodes, which is referred to as sparse wavelength conversion [8]. Thus, for a real wavelength-routed network, various wavelength conversion schemes may be deployed at different nodes. Correspondingly, the wavelength continuity constraint must be generalized to accommodate all these scenarios.

The static traffic grooming problem has been studied in [1] and [19]. Integer linear programming (ILP) formulations were presented to optimize resource cost or maximize network throughput when a fixed traffic pattern is given. Since Internet traffic is inherently dynamic, both [12] and [13] proposed grooming heuristic algorithms for the dynamic traffic grooming problem without considering the generalized wavelength continuity (GWC) constraint. The layered auxiliary graph (layered-AG) model proposed in [14] and [15] can be used to address the GWC constraint. Similar to the approach in [16], the layered-AG model has one layer for each wavelength. As a result, it has $W+2$ layers including the lightpath and access layers and each layer has $2 \times N$ nodes, where $N$ is the number of nodes in the network and $W$ is the number of wavelengths supported on each fiber link. The total number of nodes in the auxiliary graph is $2 \times N \times(W+2)$. This approach expresses the GWC constraint in the structure of the auxiliary graph. Therefore, an ordinary shortest path algorithm such as Dijkstra's algorithm can be used as the grooming algorithm. The worst case time complexity of the Dijkstra's algorithm is $\mathrm{O}\left(|V|^{2}\right)$, where $|V|$ is the number of nodes in the graph. Thus, for a Dijkstra based grooming algorithm without considering additional constraints, the worst case time complexity would be $\mathrm{O}\left(N^{2} W^{2}\right)$. In a dense WDM (DWDM) optical network, a pair of nodes may be connected by tens of fibers and each fiber may contain hundreds of wavelengths. As the size of the auxiliary graph increases linearly with the number of wavelengths, this approach may cause scalability problem for the grooming algorithm.

In this paper, we introduce a simplified auxiliary graph using the link bundling concept. All $W$ wavelengths are packed into a single physical layer in the auxiliary graph. In addition, the GWC constraint is not represented directly in the structure of the auxiliary graph. Instead, it is expressed as a constraint on the path. The grooming algorithm then becomes a constraint based routing problem. Its goal is to find the shortest path in the auxiliary graph subject to the constraint. The weights of the edges in the auxiliary graph are determined by grooming policies. To solve the constrained dynamic traffic grooming (CDTG) problem, we propose the SAG-LB method in Section IV-C to find a feasible path and assign wavelengths for a new lightpath. With this method, we propose the constrained integrated grooming algorithm (CIGA). We further propose the least resource path first (LR) grooming policy, which integrates the wavelength and transceiver metrics together. We compare the LR grooming policy with two existing grooming policies from the literature.

The rest of the paper is organized as follows. Section II introduces an integrated grooming architecture. Section III states a formal description of the CDTG problem. Section IV introduces the link bundled auxiliary graph (LBAG) model and describes the SAG-LB method to solve GWC constraint problem. Section V presents the CIGA grooming algorithm based on the LBAG model. Section VI presents the grooming policies, including two existing grooming policies from the literature and the LR grooming policy we proposed. Section VII describes the numerical results of our simulation. Section VIII concludes the paper. The Appendix provides the proofs of the theorems presented in Section IV-B.

\section{GROOMING ARCHITECTURE}

In this section, we first introduce two grooming approaches based on an overlay model and a peer model [9], respectively. Then, we describe the integrated grooming architecture used in this paper. Finally, we describe the support of grooming functionality from generalized multiprotocol label switching (GMPLS) [10] technologies.

\section{A. Grooming Approaches}

Operationally, the two layers involved in the traffic grooming can be managed separately or jointly, corresponding to an overlay or a peer deployment model, respectively. For the overlay model, the routing of lightpaths over the physical topology and the routing of connections over the virtual topology are managed by two control planes. Therefore, the client layer (the virtual topology layer) can only see lightpaths provided by the server layer (the physical topology layer). The internal structure of the physical topology is invisible to the client layer control plane. For the peer model, the two layers are managed by a single control plane. Therefore, the control plane has all the information about the two layers and the routing decisions of lightpath and connections can be considered jointly.

Depending on the deployment models, there are generally two approaches to address the traffic grooming problem. For the overlay model, the routing decisions in the two layers are considered independently. Each layer has its own routing algorithms. Specifically, the lightpath provisioning problem in the physical topology is studied as routing and wavelength assignment (RWA) problem in the literature. For the peer model, an integrated grooming algorithm is needed in the control plane to provision both lightpaths and connections. 


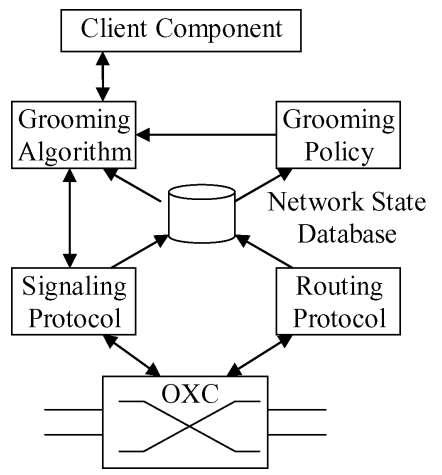

Fig. 2. Integrated grooming architecture.

\section{B. Integrated Grooming Architecture}

In this paper, we assume the integrated grooming approach (the peer model). Fig. 2 shows the essential components involved in the integrated grooming architecture. The client component refers to the entity generating connection requests to the grooming algorithm. The grooming algorithm tries to select a path for each request based on the current state of the network and grooming policy. If the grooming algorithm successfully finds a path, it will invoke the signaling protocol component to set up the connection for this request. If necessary, new lightpaths may be established before the setup of the connection. The routing protocol collects link state information from other nodes and broadcasts the local state information to the network. The OXC component must support wavelength and subwavelength switching. Depending on the implementation, the two-level switching OXC can be physically separate or integrated. Whatever the hardware implementation, the integrated grooming architecture assumes the two-level switching is managed by a uniform control plane.

\section{Grooming With GMPLS}

GMPLS [10] technologies are expected to serve as the common control plane of the next-generation optical networks. GMPLS extends multiprotocol label switching (MPLS) [11] to support time-division (e.g., SONET/SDH), wavelength, waveband, and spatial (e.g., fiber) switching. In GMPLS, the channels at different granularities such as SONET circuits, lightpaths, etc., are uniformly named as label switched paths (LSPs). To maintain a flexible LSP hierarchy, GMPLS supports nested LSP (LSP within LSP), which allows lower rate LSPs to be nested in a higher rate LSP. With the nested LSP concept, GMPLS supports the traffic grooming function by nesting subwavelength LSPs such as SONET circuits onto lightpath LSPs.

As with MPLS, GMPLS extends traditional interior gateway routing protocols (IGPs) such as OSPF and IS-IS to support constraint-based routing (CBR). The constraints for CBR calculation can be resource-related, performance-related, or administrative in nature. Specifically, the GWC constraint can be classified as a resource-related constraint for GMPLS routing calculation. Once the path of an LSP is found, the LSP can be established using one of the signaling protocols extended from CR-LDP and RSVP-TE.

\section{PRoblem DEFINITION}

In this section, the CDTG problem considered in this paper is formulated in terms of inputs, constraints, and objective function.

\section{A. Inputs}

1) Physical topology represented as a unidirectional graph $G_{p}=\left(V_{p}, E_{p}\right)$, where $V_{p}$ is a set of network nodes and $E_{p} \subset V_{p} \times V_{p}$ is the set of fiber links connecting the nodes. The number of nodes is $N=\left|V_{p}\right|$.

2) The set of wavelengths supported by each fiber is $W$ and the capacity of each wavelength is $\mathrm{C}$. We assume that the same set of wavelengths is deployed on every link. The capacity of a wavelength is normalized to an integer $\mathrm{C}$ based on the smallest grooming granularity in the network. For example, if one wavelength supports an OC-48 channel, and the smallest grooming granularity is OC- 3 , then $\mathrm{C}$ equals $48 / 3=16$.

3) The number of transmitter and receiver pairs at each node is $\Delta_{i}(1 \leq i \leq N)$. In this study, we assume the transceivers are tunable to any wavelength operating on the fiber.

4) The wavelength conversion capability in the network is represented as a function $f_{\mathrm{wc}}: W \times W \times V \rightarrow\{0,1\}$, where $f_{\mathrm{wc}}\left(\lambda_{1}, \lambda_{2}, u\right)=1$ if and only if wavelength $\lambda_{1}$ can be converted to wavelength $\lambda_{2}$ at node $u$. Specially, for a node $u$ without wavelength conversion capability, $f_{\mathrm{wc}}\left(\lambda_{1}, \lambda_{2}, u\right)=1$ if and only if $\lambda_{1}=\lambda_{2}$; for a node $u$ with full wavelength conversion capability, $f_{\mathrm{wc}}\left(\lambda_{1}, \lambda_{2}, u\right)=1 \forall \lambda_{1}, \lambda_{2} \in W$.

5) Virtual topology represented as a unidirectional graph $G_{v}=\left(V_{v}, E_{v}\right)$, where $V_{v}=V_{p}$, and $E_{v} \subset V_{v} \times V_{v}$ is the set of lightpaths connecting the nodes. Initially, $E_{v}=\varnothing$. An important assumption about the wavelength-routed network is that the network is reconfigurable, that is, the virtual topology can be dynamically changed to best serve the current set of connection requests. In this paper, we adapt an automatically gradual evolution strategy for the virtual topology. New lightpaths are established only when a new connection request is coming and needs these new lightpaths in its path. On the other hand, when a connection departures, some lightpaths may left not used by any connections. These unused lightpaths are to be torn down.

6) The set of connection rates (granularities) supported in the network is $X$. Dynamic traffic demand $(\Psi)$ is represented as a sequence of connection requests $\varphi(s, d, t, \Delta t, x)$, where $s$ is source node, $d$ is destination node, $t$ is the arrival time of the connection request, $\Delta t$ is the required service time for this request, and $x \in X$ is the connection rate in normalized traffic unit.

\section{B. Constraints}

1) Wavelength Constraint: Let $W_{a}(u, v)$ be the set of available wavelengths on the edge $(u, v)$. To establish a lightpath on a path $p, W_{a}(u, v) \neq \varnothing$ must be true for any edge $(u, v)$ in the path $p$. 
2) Transceiver Constraint: To establish a lightpath from node $u$ to $v$, node $u$ must have an available transmitter and node $v$ must have an available receiver.

3) Generalized Wavelength Continuity Constraint: Define $W_{c}(\Lambda, u)$ as the set of wavelengths that can be converted from a wavelength set $\Lambda$ at the node $u$ and $W_{r}(\Lambda, u)$ as the set of wavelengths that can be converted to one of wavelengths in set $\Lambda$ at the node $u . W_{c}(\Lambda, u)$ and $W_{r}(\Lambda, u)$ can be calculated using (1) and (2), respectively

$$
\begin{aligned}
& W_{c}(\Lambda, u)=\bigcup_{\lambda_{1} \in \Lambda}\left\{\lambda_{2} \mid \lambda_{2} \in W \text { and } f_{\mathrm{wc}}\left(\lambda_{1}, \lambda_{2}, u\right)=1\right\} \\
& W_{r}(\Lambda, u)=\bigcup_{\lambda_{2} \in \Lambda}\left\{\lambda_{1} \mid \lambda_{1} \in W \text { and } f_{\mathrm{wc}}\left(\lambda_{1}, \lambda_{2}, u\right)=1\right\} .
\end{aligned}
$$

For a path $p\left(v_{0}, v_{1}, \ldots, v_{k}\right)$, define $\lambda_{i}$ as the assigned wavelength for edge $\left(v_{i-1}, v_{i}\right)(1 \leq i \leq k)$. The GWC constraint is said to be satisfied if and only if the following conditions are met for the wavelength $\lambda_{i}(1 \leq i \leq k)$ :

$$
\begin{array}{ll}
\lambda_{i} \in W_{a}\left(v_{i-1}, v_{i}\right), & \text { for } 1 \leq i \leq k \\
\lambda_{i} \in W_{c}\left(\left\{\lambda_{i-1}\right\}, v_{i-1}\right), & \text { for } 2 \leq i \leq k .
\end{array}
$$

4) Lightpath Capacity Constraint: The total bandwidth of all the connections carried over a lightpath must not be larger than the bandwidth of a lightpath.

\section{Objective}

The objective of the CDTG problem is to maintain a dynamically changing virtual topology and find a path subject to all the constraints in the virtual topology for each connection request. The path may include both existing and new lightpaths. If it has new lightpaths, then new lightpaths must be established before the connection can be set up. If no path can be found for this request, it is blocked. The solution can be defined as a function $s: \Psi \rightarrow\{0,1\}$, where, for each request $\varphi \in \Psi, s(\varphi)=1$ if and only if $\varphi$ is satisfied. If $v(\varphi)$ is the associated value of the request $\varphi$, the optimal solution to this problem is to define the function $s(\varphi)$ so that the weighted sum of all the successfully routed traffic $w s t(\Psi)$ as defined in (5) is maximized

$$
w s t(\Psi)=\sum_{\varphi \in \Psi} v(\varphi) \times s(\varphi)
$$

where $v(\varphi)$ is the associated value of the request $\varphi$. Depending on the scenario, $v(\varphi)$ can be defined as the bandwidth of the request $\varphi$, the product of its bandwidth, and holding time, etc.

This global optimal objective can possibly be achieved only if all the connection requests are known in advance. In that case, some earlier requests may need to be blocked to satisfy more valuable later requests, even though the earlier requests can be satisfied at the time they arrive. However, in a dynamic environment, the earlier requests cannot have any knowledge of the later requests. Therefore, the grooming algorithm has to try its best to satisfy each arriving request. In this case, the grooming algorithm can only count on the grooming policy to influence the path selection of each request. It is expected that a good grooming policy would use the network resource efficiently and generate an overall near-optimal solution.
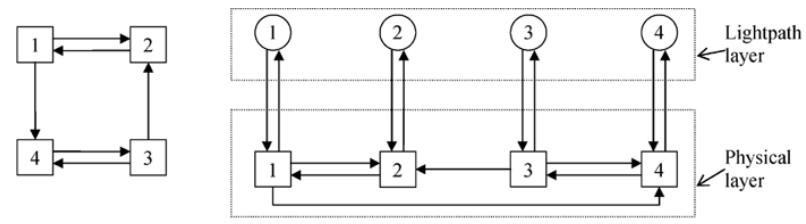

(a)

(b)

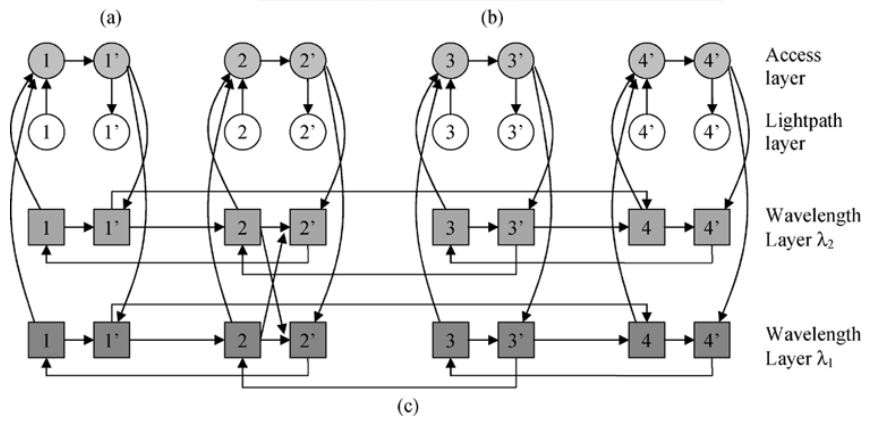

Fig. 3. (a) Physical topology of a four-node sample network. Each fiber link supports two wavelengths. Node 2 has full wavelength conversion capability. (b) LBAG. (c) Layered-AG.

\section{LinK BundLED AuXILIARY GRAPH (LBAG) MODEL}

In this section, we first present our LBAG model, and then describe the method used to solve the GWC constraint in the LBAG model.

\section{A. Link Bundled Auxiliary Graph (LBAG)}

Link bundling is a concept introduced in GMPLS to assist the management of a network with an excessive number of links between two adjacent nodes. For link-state routing protocols, if link bundling is not used, the information about each wavelength has to be advertised separately in the network. This is a huge traffic overhead and burdens routing protocols. With link bundling, links with the same properties can be advertised as a single bundled link in the routing protocols. Link bundling techniques reduce the amount of link state information that has to be advertised by the routing protocols and, therefore, improves the scalability of routing protocols.

Using the link bundling concept, the layered-AG in earlier work [14]-[16] (described in Section I) can be largely simplified. For a network with physical topology $G_{p}=\left(V_{p}, E_{p}\right)$, the LBAG $G_{a}=\left(V_{a}, E_{a}\right)$ is constructed as a two-layered graph with $\left|V_{p}\right|$ nodes in each layer. The two layers are called the physical layer and the lightpath layer, respectively. For each node in the network, there are two nodes, one in each layer, in the auxiliary graph. The two nodes are called the physical node and the virtual node, respectively.

The edges in the physical layer represent the links in the physical topology and the edges in the lightpath layer represent the lightpaths in the virtual topology. In addition, the edges between the lightpath layer and physical layers represent transceiver resource. Fig. 3(b) shows an example of the LBAG model for a four-node sample network, as shown in Fig. 3(a). For comparison reasons, Fig. 3(c) shows how the layered-AG [14] would look like for the same sample network.

The three categories of edges in the LBAG are constructed as follows.

1) Wavelength Edges $\left(E_{w}\right)$ : For a link from node $u$ to $v$ in the physical topology, there is a wavelength edge from physical 


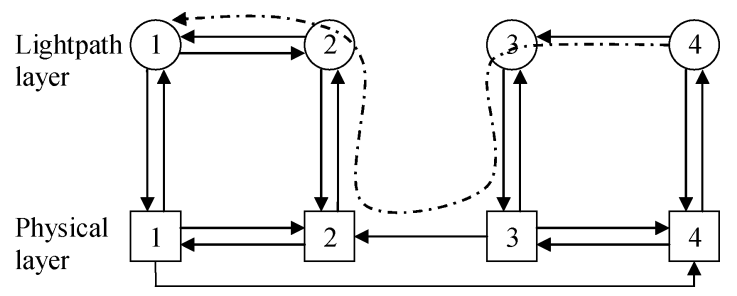

Fig. 4. Path in the LBAG.

node $u$ to $v$ in the auxiliary graph. No matter how many fibers and wavelengths in a single link, a single wavelength edge is used in the auxiliary graph. At operation time, if no wavelength is available on a link, the corresponding wavelength edge is removed from the auxiliary graph or alternatively its metric is set to $\infty$.

2) Lightpath Edges $\left(E_{l}\right)$ : For each lightpath from node $u$ to $v$ in the virtual topology, there is a lightpath edge from virtual node $u$ to $v$ in the auxiliary graph. For a specific connection request, if the available bandwidth on a lightpath is smaller than the bandwidth requirement of the request, the lightpath edge may be temporarily removed or alternatively its metric is set to $\infty$.

3) Transceiver Edges: Transceiver edges are connecting the lightpath layer and physical layer in the auxiliary graph. They represent the transceiver resource in the network. Transceiver edges can be further divided into transmitter edges $\left(E_{t}\right)$ and receiver edges $\left(E_{r}\right)$. For a node $u$, if there are transmitters available, then there is a transmitter edge from virtual node $u$ to physical node $u$ in the auxiliary graph. On the other hand, if there are receivers available on this node, then there is a receiver edge from physical node $u$ to virtual node $u$ in the auxiliary graph.

A valid path in the LBAG should always begin and end in the lightpath layer. Once the path enters the physical layer through a transmitter edge, it implies a new lightpath will be established. The source node of the new lightpath is the node whose transmitter the transmitter edge represents. Finally, the path will enter the lightpath layer again through a receiver edge. The node whose receiver the receiver edge represents is the destination node of the new lightpath. A path may have multiple subpaths alternating in the physical layer and lightpath layer. Therefore, multiple new lightpaths may need to be established. Fig. 4 shows a path from node 4 to node 1 in the auxiliary graph. If a connection is to be routed on this path, a new lightpath from node 3 to node 2 must be established first. Then, the connection is routed on the three-hop path in the virtual topology.

\section{B. Generalized Wavelength Continuity Constraint}

To find a path in the LBAG for a connection, we must ensure that the subpaths in the physical layer satisfy the GWC constraint. This section proves several theorems which can be used to solve this problem. Specifically, a path in this section refers to a subpath in the physical layer of a LBAG.

Definition 1: For a path $p\left(v_{0}, v_{1}, \ldots, v_{k}\right)$, define path forward compatible wavelength set (PFC-WS) $W_{f}\left(p, v_{i}\right)(0 \leq i \leq$ $k$ ) as (6), which calculates the set of available wavelengths at the last link $\left(v_{i-1}, v_{i}\right)$ of the subpath $p_{i}\left(v_{0}, v_{1}, \ldots, v_{i}\right)$ that can be used to establish a lightpath on $p_{i}$ when the calculation proceeds in the forward direction from the source node $v_{0}$. For simplicity, $W_{f}(p)$ is used to represent $W_{f}\left(p, v_{k}\right)$

$$
\begin{aligned}
& W_{f}\left(p, v_{i}\right) \\
& =\left\{\begin{array}{ll}
W, & \text { if } i=0 \\
W_{c}\left(W_{f}\left(p, v_{i-1}\right), v_{i-1}\right) \cap W_{a}\left(v_{i-1}, v_{i}\right), & \text { if } 1 \leq i \leq k
\end{array} .\right.
\end{aligned}
$$

Definition 2: For a path $p\left(v_{0}, v_{1}, \ldots, v_{k}\right)$, define path backward compatible wavelength set (PBC-WS) $W_{b}\left(p, v_{i}\right)(0 \leq i \leq$ $k$ ) as (7), which calculates the set of available wavelengths at the first link $\left(v_{i}, v_{i+1}\right)$ of the subpath $p_{i}\left(v_{i}, v_{i+1}, \ldots, v_{k}\right)$ that can be used to establish a lightpath on $p_{i}$ when the calculation proceeds in the backward direction from the destination node $v_{k}$. For simplicity, $W_{b}(p)$ is used to represent $W_{b}\left(p, v_{0}\right)$

$$
\begin{aligned}
& W_{b}\left(p, v_{i}\right) \\
& =\left\{\begin{array}{ll}
W, & \text { if } i=k \\
W_{r}\left(W_{b}\left(p, v_{i+1}\right), v_{i+1}\right) \cap W_{a}\left(v_{i}, v_{i+1}\right), & \text { if } 0 \leq i \leq k-1
\end{array} .\right.
\end{aligned}
$$

Theorem 1: For a path $p\left(v_{0}, v_{1}, \ldots, v_{k}\right)$, if $W_{f}\left(p, v_{k}\right) \neq \varnothing$, then (8) gives a feasible wavelength assignment satisfying the GWC constraint.

$$
\lambda_{i}=\left\{\begin{array}{ll}
\forall \lambda \in W_{f}\left(p, v_{k}\right), & \text { if } i=k \\
\forall \lambda \in\left(W_{r}\left(\left\{\lambda_{i+1}\right\}, v_{i}\right) \cap W_{f}\left(p, v_{i}\right)\right), & \text { if } 1 \leq i \leq k-1
\end{array} .\right.
$$

Theorem 2: For a path $p\left(v_{0}, v_{1}, \ldots, v_{k}\right)$, the GWC constraint is satisfied if and only if $W_{f}\left(p, v_{k}\right) \neq \varnothing$.

Theorem 3: For a path $p\left(v_{0}, v_{1}, \ldots, v_{k}\right)$, the GWC constraint is satisfied if and only if $W_{b}\left(p, v_{0}\right) \neq \varnothing$.

This theorem complements Theorem 2 by considering the GWC constraint from the destination node of a path.

Theorem 4: For a path $p\left(v_{0}, v_{1}, \ldots, v_{k}\right)$, the GWC constraint is satisfied if and only if $W_{c}\left(W_{f}\left(p, v_{k}\right), v_{i}\right) \cap$ $W_{b}\left(p, v_{i}\right) \neq \varnothing$ for any $0 \leq i \leq k$.

Theorem 4 is a generalized theorem of Theorems 2 and 3.

Please see the Appendix for the proofs of the Theorems 1 and 2. The proofs for Theorems 3 and 4 are similar and, hence, omitted.

\section{Simplified Auxiliary Graph With Link Bundling (SAG-LB) Method}

With the above theorems, we propose a simplified auxiliary graph with link bundling (SAG-LB) method to solve the GWC constraint problem for subpaths in the physical layer. The SAG-LB method has two steps.

Step 1) Find a feasible path satisfying all the constraints. For the GWC constraint, we can calculate the PFC-WS (see Definition 1) once the path search enters the physical layer. If PFC-WS is not empty for the last wavelength edge, then by Theorem 2, we know that a feasible wavelength assignment is available for the current subpath in the physical layer. If PFC-WS is empty for the last wavelength edge, then this path can be safely removed from the search space since it is not possible to find a feasible wavelength assignment on this subpath. 


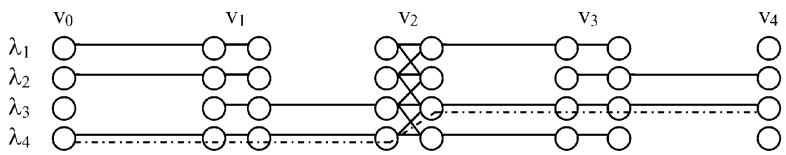

(a)

$\mathrm{W}_{\mathrm{a}=\left\{\lambda_{1}, \lambda_{2}, \lambda_{4}\right\}}^{\mathrm{W}_{\mathrm{a}}=\left\{\lambda_{3}, \lambda_{4}\right\}} \mathrm{W}^{\mathrm{W}_{\mathrm{a}}=\left\{\lambda_{1}, \lambda_{3}, \lambda_{4}\right\}} \mathrm{O}^{\mathrm{W}_{\mathrm{a}}=\left\{\lambda_{2}, \lambda_{3}\right\}} \mathrm{O}$

$\mathrm{W}_{\mathrm{f}}\left(\mathrm{v}_{1}\right)=\left\{\lambda_{1}, \lambda_{2}, \lambda_{4}\right\} \quad \mathrm{W}_{\mathrm{f}}\left(\mathrm{v}_{2}\right)=\left\{\lambda_{4}\right\} \quad \mathrm{W}_{\mathrm{f}}\left(\mathrm{v}_{3}\right)=\left\{\lambda_{3}, \lambda_{4}\right\} \quad \mathrm{W}_{\mathrm{f}}\left(\mathrm{v}_{4}\right)=\left\{\lambda_{3}\right\}$

$\mathrm{W}_{\mathrm{f}}\left(\mathrm{v}_{4}\right)=\left\{\lambda_{3}\right\} \neq \varnothing \rightarrow$ GWC constraint is satisfied on this path.

Wavelength Assignment:

For edge $\left(\mathrm{v}_{3}, \mathrm{v}_{4}\right), \mathrm{W}_{\mathrm{f}}\left(\mathrm{v}_{4}\right)=\left\{\lambda_{3}\right\} \rightarrow \lambda_{\mathrm{v} 3 \mathrm{v} 4}=\lambda_{3}$

For edge $\left(\mathrm{v}_{2}, \mathrm{v}_{3}\right), \mathrm{W}_{\mathrm{c}}\left(\lambda_{\mathrm{v} 3 \mathrm{v} 4}, \mathrm{v}_{3}\right)=\left\{\lambda_{3}\right\} \rightarrow \mathrm{W}_{\mathrm{c}}\left(\lambda_{\mathrm{v} 3 \mathrm{v} 4}, \mathrm{v}_{3}\right) \cap \mathrm{W}_{\mathrm{f}}\left(\mathrm{v}_{3}\right)=\left\{\lambda_{3}\right\} \rightarrow \lambda_{\mathrm{v} 2 \mathrm{v} 3}=\lambda_{3}$

For edge $\left(\mathrm{v}_{1}, \mathrm{v}_{2}\right), \quad \mathrm{W}_{\mathrm{c}}\left(\lambda_{\mathrm{v} 2 \mathrm{v} 3}, \mathrm{v}_{2}\right)=\left\{\lambda_{2}, \lambda_{3}, \lambda_{4}\right\} \rightarrow \mathrm{W}_{\mathrm{c}}\left(\lambda_{\mathrm{v} 2 \mathrm{v} 3}, \mathrm{v}_{2}\right) \cap \mathrm{W}_{\mathrm{f}}\left(\mathrm{v}_{2}\right)=\left\{\lambda_{4}\right\} \rightarrow \lambda_{\mathrm{v} 1 \mathrm{v} 2}=\lambda_{4}$

For edge $\left(\mathrm{v}_{0}, \mathrm{v}_{1}\right), \quad \mathrm{W}_{\mathrm{c}}\left(\lambda_{\mathrm{v} 1 \mathrm{v} 2}, \mathrm{v}_{1}\right)=\left\{\lambda_{4}\right\} \rightarrow \mathrm{W}_{\mathrm{c}}\left(\lambda_{\mathrm{v} 1 \mathrm{v} 2}, \mathrm{v}_{1}\right) \cap \mathrm{W}_{\mathrm{f}}\left(\mathrm{v}_{1}\right)=\left\{\lambda_{4}\right\} \rightarrow \lambda_{\mathrm{v} 0 \mathrm{v} 1}=\lambda_{4}$

(b)

Fig. 5. Illustration of the methods to solve GWC constraint in the auxiliary graph models. (a) Conventional layered-AG model. (b) LBAG model. $\left(v_{0}\right.$, $v_{1}, v_{2}, v_{3}, v_{4}$ ) is a path in the physical topology. Suppose nodes $v_{1}$ and $v_{3}$ do not have conversion capability and node $v_{2}$ has limited-range wavelength conversion capability to convert wavelength $\lambda_{i}$ into $\lambda_{i-1}$ and $\lambda_{i+1}$. Note that only physical (or wavelength) layers are shown in this figure for simplicity.

Step 2) Once a feasible path is selected, we can find a feasible wavelength assignment for each physical layer subpath contained in this path using (8) in Theorem 1 . If there is more than one wavelength which can be assigned for an edge by (8), then any wavelength assignment heuristic such as first-fit, random, most-used heuristics [20] can be used to select a wavelength.

Fig. 5 illustrates the SAG-LB method for a subpath in the physical layer, where each fiber supports four wavelengths. Fig. 5(a) shows the conventional approach to represent each wavelength separately and express wavelength conversion capability using $2 \times W(W=4)$ nodes for each network node. Fig. 5(b) shows the process of applying the SAG-LB method. First, we calculate $W_{f}$ for each edge. By Theorem 1, $W_{f}\left(p, v_{4}\right) \neq \varnothing$ implies that the GWC constraint is satisfied. If all the other constraints are satisfied, we can assign wavelength for each edge using (8) in Theorem 1 . The wavelength assignment starts from the last edge $\left(v_{3}, v_{4}\right)$ and ends with the first edge $\left(v_{0}, v_{1}\right)$. Specially, the calculation in Fig. 5 shows there is exactly one wavelength that can be used on each edge, which is $\lambda_{3}$ on edge $\left(v_{3}, v_{4}\right), \lambda_{3}$ on edge $\left(v_{2}, v_{3}\right), \lambda_{4}$ on edge $\left(v_{1}, v_{2}\right)$, and $\lambda_{4}$ on edge $\left(v_{0}, v_{1}\right)$.

\section{GROOMING ALGORITHM}

In this section, we propose the constrained integrated grooming algorithm (CIGA) based on the LBAG model. We also propose a constrained grooming shortest-path (CGSP) algorithm which is used as a procedure in CIGA. The CGSP algorithm calculates the shortest path available in the LBAG for a connection request.

\section{A. Constrained Integrated Grooming Algorithm (CIGA)}

While the CIGA grooming algorithm shown in Fig. 6 only describes grooming procedure for arriving connection requests, the grooming procedure for leaving connections is simpler. It
Input: $G_{p}$, physical topology; $w$, weight function decided by the grooming policy; and connection requests.

1 Initialize the LBAG $G_{a}$ according to the physical topology. The initial edge metrics are decided by the specific grooming policy used.

2 loop for each arriving connection request $(s, d, x)$. if (grooming policy is adaptive)

Update the metrics of all the edges.

$p \leftarrow \operatorname{CGSP}\left(G_{a}, w, s, d, x\right)$

if $(p)$

for each physical layer sub-path $p_{s}$ in $p$ do Assign a wavelength for each link of $p_{s}$ using equation (8) defined in theorem 1.

Establish a new lightpath on $p_{s}$.

Add a lightpath edge in $G_{a}$ accordingly and

determine its initial metric by the grooming policy.

Update the metrics of the wavelength and transceiver edges involved in the new lightpath.

Set up a connection for the request on path $p$ using the existing or new lightpaths.

Update the metrics of the lightpath edges involved in the connection.

else

Block the request.

Fig. 6. CIGA grooming algorithm.

disconnects the connection and releases the bandwidth on lightpaths it used. If a lightpath is not used by any other connections, it disconnects the lightpath as well and releases the wavelength and transceiver resources allocated for this lightpath. It updates the metrics of the relevant edges in the LBAG accordingly.

\section{B. Constrained Grooming Shortest-Path (CGSP) Algorithm}

The proposed CGSP algorithm is used in the above CIGA grooming algorithm to calculate the shortest available path in the auxiliary graph. CGSP is a constraint-based shortest-path algorithm which extends Dijkstra' algorithm [21] to address the GWC constraint in the physical topology. The techniques of $k$-shortest paths [17] and dominated path pruning (DPP) [18] are used in CGSP along with the SAG-LB method when the path searching process enters the physical layer. As only the physical layer is subject to the GWC constraints, for all the nodes in the virtual layer, the regular relaxation procedure [21] of Dijkstra's algorithm suffices.

1) Constrained $k$-Shortest Paths: As we know, as an outcome of calculating the shortest path from a source node $s$ to a destination node $d$, Dijkstra's algorithm also obtains the shortest paths from source node $s$ to all the intermediate nodes. In CGSP, in order to calculate the shortest path from a source node $s$ to a destination node $d$ in the physical layer, $k$-shortest paths from the source node $s$ to the intermediate nodes need to be calculated. This is because the subpaths within a shortest path subject to the GWC constraint are not necessarily the shortest subpaths, as illustrated in Fig. 7. There are three subpaths from $u$ to $v$ in the physical layer and suppose node $v$ does not have wavelength conversion capability. If only the shortest path, which is $p_{1}$, is explored, then the algorithm cannot find a feasible path from $u$ to $w$ because of no common available wavelength between $p_{1}$ and $p_{4}$. On the other hand, if the algorithm explores $k(k>1)$ shortest paths, then another path $p_{3}$ from $u$ to $v$ can be used with 


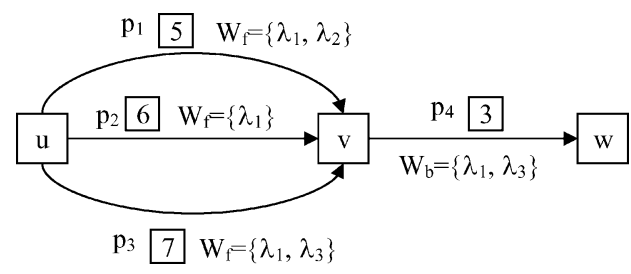

Fig. 7. Illustration of the necessity of exploring $k$-shortest paths for intermediate physical nodes.

path $p_{4}$ to form a feasible shortest path from $u$ to $w$ on wavelength $\lambda_{3}$.

Note that CGSP does not check the GWC constraint on a path by path basis after the $k$-shortest paths from the source to destination are found. Instead, it embeds the GWC constraint checking into the $k$-shortest path relaxation process, as shown in Fig. 8. At the intermediate physical nodes between the source and destination nodes, the $k$-shortest subpaths from the source node satisfying the GWC constraints are calculated. Eventually, only the shortest path from the source node to the destination node satisfying all the constraints will be returned by the CGSP algorithm.

2) Dominated-Path Pruning (DPP): A path $p$ is said to be dominated by another path $q$ if the length (or cost) of $p$ is larger or equal to that of $q$ and $W_{f}(p) \subseteq W_{f}(q)$, with at least one inequality for the two conditions. For example, in Fig. 7, path $p_{2}$ is dominated by path $p_{1}$, because the length of $p_{2}(6)$ is larger than that of $p_{1}(5)$ and $W_{f}\left(p_{2}\right)=\left\{\lambda_{3}\right\} \subset\left\{\lambda_{1}, \lambda_{3}\right\}=W_{f}\left(p_{1}\right)$. The concept of dominated-path is used to reduce search space without compromising the search result. While the dominatedpaths are pruned, only nondominated-paths are stored at each node and possibly used as subpaths for longer paths.

3) CGSP Algorithm Description: The CGSP algorithm is shown in Fig. 8. Initially, all the nodes in the auxiliary graph are in white color. The counter $s p c[u]$ records the number of $k$-shortest paths that have been determined at node $u$. For virtual nodes, $k=1$; for physical nodes, $k \geq 1$. Once all $k$-shortest paths from the source have been determined for a node $u$, it is colored black. Otherwise, it is colored gray if at least the shortest path from the source has been determined. The counter $p c[u]$ records the number of paths stored at node $u$, though these stored paths are not necessarily the $k$-shortest paths. Initially, the stored paths are in gray color. The set $Q$ contains all the stored gray paths in the graph which have not been identified as one of $k$-shortest paths for any node. The function $\operatorname{Extract-Min}(Q)$ finds the shortest-path $(u, i)$ in set $Q$. This path is identified as one of the $k$-shortest paths for node $u$ and is colored black. For each adjacent node $v$ of node $u$, if it is not in the path $(u, i)$ and its color is not in black, the edge $(u, v)$ is relaxed.

In the RELAX procedure, if $v$ is a virtual node and if a shorter path from the source to node $v$ is found by going through $u$ to $v$, then the stored path at node $v$ is updated. For a physical node $v$, on the other hand, the PFC-WS for edge $(u, v)$ is computed first to make sure it is not empty. If the PFC-WS is not empty, then check whether this path is a dominated path by the current stored paths at node $v$. If it is not dominated by any paths stored at node $v$, then remove those stored paths which are dominated by this newly found path, if those paths exist. Because only the

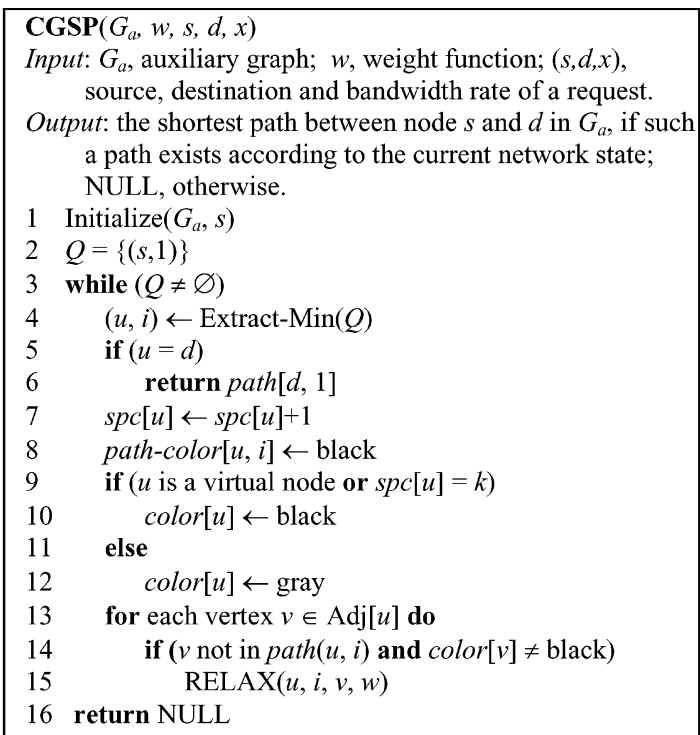

$\operatorname{RELAX}(u, i, v, w)$

if ( $v$ is a virtual node)

if $(c[v, 1]>c[u, i]+w(u, v))$

$c[v, 1] \leftarrow c[u, i]+w(u, v)$

else

$\operatorname{path}[v, 1] \leftarrow \operatorname{path}[u, i]+(u, v)$

if ( $u$ is a virtual node)

$W_{t} \leftarrow W$

else

$W_{t} \leftarrow W_{c}\left(W_{f}(u, i), u\right) \cap W_{a}(u, v)$

if $\left(W_{t} \neq \varnothing\right)$

$\operatorname{cost} \leftarrow c[u, i]+w(u, v)$

if (Non-Dominated-Path $\left(v, W_{t}\right.$, cost $\left.)\right)$

Del-Dominate-Path $\left(v, W_{t}\right.$, cost $)$

if $(p c[v]<k)$

Insert-Path $\left(u, i, v, W_{t}, \cos t\right)$

else

Replace-Path $\left(u, i, v, W_{t}\right.$, cost $)$

Fig. 8. CGSP algorithm.

$k$-shortest paths needs to be identified, if there are less than $k$ paths found for node $v$, then add the newly found path; otherwise, replace the longest path stored at node $v$ if that path is longer than the newly found path. The lines 12 and 13 can be omitted if we do not apply DPP to this algorithm.

\section{Worst Case Time Complexity Analysis}

In the RELAX procedure, the Insert-Path operation takes $\mathrm{O}(1)$ time and the replace-path operation takes $\mathrm{O}(k)$ time. Both Nondominated-Path and Del-Dominate-Path operations takes $\mathrm{O}\left(k W^{2}\right)$ time. The lines 6-7 of RELAX take $\mathrm{O}\left(W^{2}\right)$ time. Therefore, the worst case time complexity of RELAX operation with DPP is $\mathrm{O}\left(k W^{2}\right)$, otherwise, it is $\mathrm{O}\left(W^{2}\right)$.

Let $N$ be the number of nodes in the optical network. Then, there are $N$ virtual nodes and $N$ physical nodes in the LBAG. In 
the CGSP algorithm, the Initialize operation of line 1 executes $2 N$ times, one for each node. As there are at most $k N+N$ paths in the $Q$, while the loop of lines 3-15 execute at most $k N+N$ times. The running time of Extract-Min operation depends on the implementation of $Q$. For a normal array or linked list implementation, the Extract-Min operation takes $\mathrm{O}(k N)$ time as it has to go through $Q$ to find the shortest path. In the worst case, the for loop of lines 13-15 executes $k\left|E_{w}\right|+\left|E_{l}\right|+\left|E_{t}\right|+\left|E_{r}\right|$ times, because each wavelength edge in the physical layer may be relaxed at most $k$ times and the other edges can be relaxed only once. While $\left|E_{t}\right|=\left|E_{r}\right|=N,\left|E_{w}\right|$ and $\left|E_{l}\right|$ are usually larger than $N$. Therefore, the for loop takes $\mathrm{O}\left(k\left|E_{w}\right|+\left|E_{l}\right|\right)$ time. The worst case time complexity of the CGSP algorithm is

$$
\begin{aligned}
\mathrm{O}(k N) \times \mathrm{O}(k N)+\mathrm{O} & \left(k\left|E_{w}\right|+\left|E_{l}\right|\right) \times \mathrm{O}\left(k W^{2}\right) \\
& =\mathrm{O}\left(k^{2} N^{2}+k^{2}\left|E_{w}\right| W^{2}+k\left|E_{l}\right| W^{2}\right) .
\end{aligned}
$$

For the CGSP algorithm without using DPP, the time complexity is $\mathrm{O}\left(k^{2} N^{2}+k\left|E_{w}\right| W^{2}+\left|E_{l}\right| W^{2}\right)$.

For a DWDM network where $\mathrm{O}(W) \geq \mathrm{O}(N)$, the time complexity can be simplified as $\mathrm{O}\left(k^{2}\left|E_{w}\right| W^{2}+k\left|E_{l}\right| W^{2}\right)$. In practice, we can assume $k$ is a small constant number, then the time complexity can be written as $\mathrm{O}\left(\left|E_{w}\right| W^{2}+\left|E_{l}\right| W^{2}\right)$. This assumption is reasonable because the simulation in Section VII shows that a moderate $k$ can achieve almost the same blocking performance as a larger $k$. In some cases, a smaller $k$ can even yield better blocking performance than a larger $k$. As $\mathrm{O}\left(\left|E_{w}\right|\right) \leq \mathrm{O}\left(N^{2}\right)$ and $\mathrm{O}\left(\left|E_{l}\right|\right) \leq \mathrm{O}\left(N^{2}\right)$, we can obtain that $\mathrm{O}\left(\left|E_{w}\right| W^{2}+\left|E_{l}\right| W^{2}\right) \leq \mathrm{O}\left(N^{2} W^{2}\right)$, where $\mathrm{O}\left(N^{2} W^{2}\right)$ is the worst case time complexity of the grooming algorithm based on the layered-AG model in earlier studies [14], [15]. This means that the worst case time complexity of the LBAG approach is no larger than that of the layered-AG approach. Simulation results in Section VII further show that the average running time of the LBAG approach is significant smaller than that of the layered-AG approach.

\section{GRoOMING POLICIES}

As there are usually multiple feasible paths available in the network to carry a connection, the problem is how to select a path from these paths. This path preference is defined by the grooming policy. In the two-layer grooming literature, two existing grooming policies are shortest physical (SP) path first and least virtual hop (LVH) path first grooming policies. As indicated by their names, the SP grooming policy prefers the path with the shortest physical fiber length and the LVH grooming policy favors the path with the least number of virtual hops. The objectives of the two grooming policies are to achieve high utilization of wavelengths and transceivers, respectively.

In the grooming algorithm, the CGSP algorithm always selects the shortest path satisfying all the constraints to carry a connection. However, the grooming policy can be implemented by defining appropriate edge weight functions for the auxiliary graph. In this section, we first explain how to implement SP and LVH grooming policies with our grooming algorithm. Then we present a new grooming policy, namely, the least resource (LR) path first grooming policy.

\section{A. Shortest Physical (SP) Path First}

The SP grooming policy can be implemented with our grooming algorithm using the weight function defined in (9)

$$
w(e)= \begin{cases}l(e), & \text { if } e \in E_{w} \\ \sum_{e_{w} \in E_{w}(e)} l\left(e_{w}\right), & \text { if } e \in E_{l} \\ 0, & \text { if } e \in E_{t} \cup E_{r}\end{cases}
$$

where $l(e)$ is the physical fiber length of a wavelength edge $e$ and $E_{w}(e)$ is the set of wavelength edges on which the lightpath $e$ is established.

Specially, if we let $l(e)=1$ for every wavelength edge, SP becomes least physical hop (LPH) first grooming policy, where the path with LPHs is preferred.

\section{B. Least Virtual Hop (LVH) Path First}

The LVH grooming policy can be implemented with our grooming algorithm using the weight function defined in (10)

$$
w(e)=\left\{\begin{array}{ll}
0, & \text { if } e \in E_{w} \cup E_{r} \\
1, & \text { if } e \in E_{l} \cup E_{t}
\end{array} .\right.
$$

\section{Least Resource (LR) Path First}

While SP grooming policy only considers the wavelength resource in the weight function and LVH grooming policy considers transceiver resource only, LR grooming policy combines the wavelength and transceiver metrics together. For LR grooming policy, the path with the least wavelength and transceiver resources is selected. The LR grooming policy can be implemented with our grooming algorithm using the weight function defined in (11)

$$
w(e)= \begin{cases}1, & \text { if } e \in E_{w} \\ \alpha, & \text { if } e \in E_{t} \\ 0, & \text { if } e \in E_{r} \\ \alpha+h c(e), & \text { if } e \in E_{l}\end{cases}
$$

where $h c(e)$ is the number of physical links within the lightpath $e$ and $\alpha$ is a predetermined number representing the ratio of transceiver and wavelength weight. For a network, if transceiver is the relatively scarce resource, we would expect $\alpha$ to be a relatively large number so that a path with less number of lightpaths is favored. On the other hand, if wavelength is the relatively scarce resource, $\alpha$ should be small so that a path with less number of physical hops is favored.

\section{NUMERICAL RESULTS}

To evaluate the performance of the grooming algorithm and the grooming policies, we simulate them on the NSF network and the EUPAN network, as shown in Fig. 9. The NSF network has 14 nodes and 42 unidirectional links. The EUPAN network has 22 nodes and 90 unidirectional links. We also compare the proposed CIGA grooming algorithm based on the LBAG model with a grooming algorithm in [14] and [15] based on the layered auxiliary graph in terms of blocking probability and running time. 


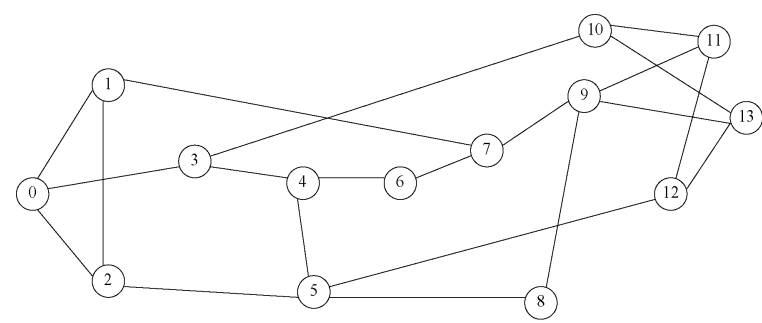

(a)

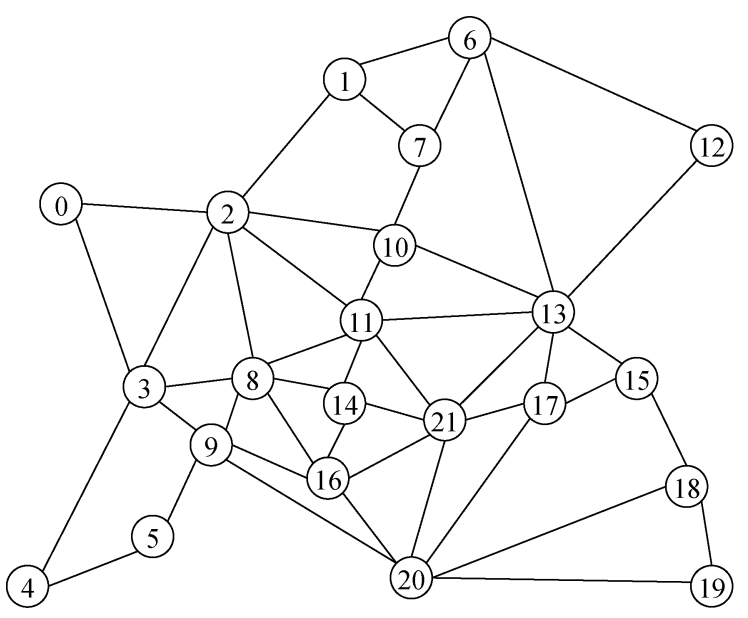

(b)

Fig. 9. Networks used in the simulation. (a) NSF network. (b) EUPAN network.

The following assumptions are used in our simulation. The arrival of connection requests on each node is a Poisson process with rate $\lambda$. The connection requests arriving at a node are uniformly destined to all the other nodes. The service time of the connection requests is exponentially distributed with unit mean. The grooming factor $C$ is 16 and the set of supported connection rates is $X=\{1,4,16\}$ after normalization. Connections at each rate request the same amount of total bandwidth, that is, the probability that an arriving connection request is at rate $x$ is $(1 / x) / \sum_{x \in X}(1 / x)$. For the NSF network, each node has 24 transmitter and receiver pairs and each fiber link supports 16 wavelengths. For the EUPAN network, each node has 36 transmitter and receiver pairs and each fiber link supports 24 wavelengths. We simulate 300000 connection requests for each scenario in this section. In addition, without explicit specification, no wavelength conversion capability is assumed.

As the connection rate varies, we calculate the traffic blocking probability (TBP) as follows:

$$
\operatorname{TBP}(\Psi)=\frac{\sum_{\varphi \in \Psi_{b}} x(\varphi)}{\sum_{\varphi \in \Psi} x(\varphi)}
$$

where $\Psi$ is the set of the connection requests, $\Psi_{b} \subseteq \Psi$ is the set of blocked connection requests, and $x(\varphi)$ is the bandwidth rate of connection request $\varphi$.

\section{A. Grooming Policy Comparison}

In this section, we compare the performance of the grooming policies in terms of TBP. The CIGA grooming algorithm with the DPP technique is used. Fig. 10 compares the performance of the LPH, LVH, and LR grooming policies. Note that $k$ is the number of shortest paths discovered for the physical nodes in the CGSP algorithm (as illustrated in Fig. 7). For LR grooming policy, the $\alpha$ parameter in (11) is fixed at 1 . As can be seen from Fig. 10, LPH performs better in a relatively sparse NSF network, where it is more difficult to find an alternate path and, thus, the wavelength resource constraint becomes more stringent. LVH, on the other hand, performs better in a relatively dense EUPAN network, where plenty of alternate paths exist and the transceiver resource constraint becomes more of a concern than the wavelength resource constraint. The LR grooming policy, which considers both the transceiver and wavelength constraints, has a good performance which is slightly below LPH in the NSF network and has the best performance among the three in the EUPAN network.

\section{B. Performance of the CIGA Grooming Algorithm}

In this section, we evaluate the performance of CIGA grooming algorithm in terms of TBP and running time when different $k$ values are applied. In addition, we investigate the effects of the DPP technique on the grooming algorithm. The LR grooming policy is used for all the scenarios in this section.

Fig. 11 shows the blocking performance of CIGA without DPP when different values of $k$ from 1 to 5 are applied. As can be seen, in both networks, the blocking probability generally decreases as $k$ increases. However, the blocking probability improvement margin drops as $k$ increases. While there is obvious improvement in blocking probability when $k$ increases from 1 to 2 and from 2 to 3 , there is hardly any improvement when $k$ increases from 4 to 5 . The weighted blocking probability does not always drop as the value of $k$ increases. In fact, in some cases, a larger $k$ may lead to a higher blocking probability. This is because a larger $k$ increases the chance of satisfying a single connection request at the expense of possibly using a more costly path in terms of resource usage. Therefore, if many connections are carried over such costly paths, the network resources are not utilized efficiently, which results in a higher blocking probability.

The DPP technique reduces the path search space by eliminating dominated-paths at intermediate nodes. It also increases the chances of satisfying connection requests because all the paths stored at intermediate nodes are not dominated by each other. This effect is illustrated in Fig. 12, which compares the blocking probabilities of CIGA using DPP (CIGA_DPP) and CIGA without using DPP (CIGA_NO_DPP). As can be seen in Fig. 12(a), CIGA_DPP using a smaller $k$ can achieve a lower blocking probability than CIGA_NO_DPP using a larger $k$. For example, CIGA_DPP with $k=2$ outperforms CIGA_NO_DPP with $k=3$. Also, CIGA_DPP with $k=3$ outperforms CIGA_NO_DPP with $k=5$. Similar results can be found in Fig. 12(b) for the EUPAN network.

DPP proves to be an effective technique to improve the blocking probability, as well as the running time, as illustrated in Fig. 13, which shows the normalized running time of CIGA with different $k$ values and under different loads. Under any given load, the actual running time of CIGA_NO_DPP with $k=1$ is assumed as 1 unit time. The normalized running times at other parameters ( $k$ and DPP) are calculated according 


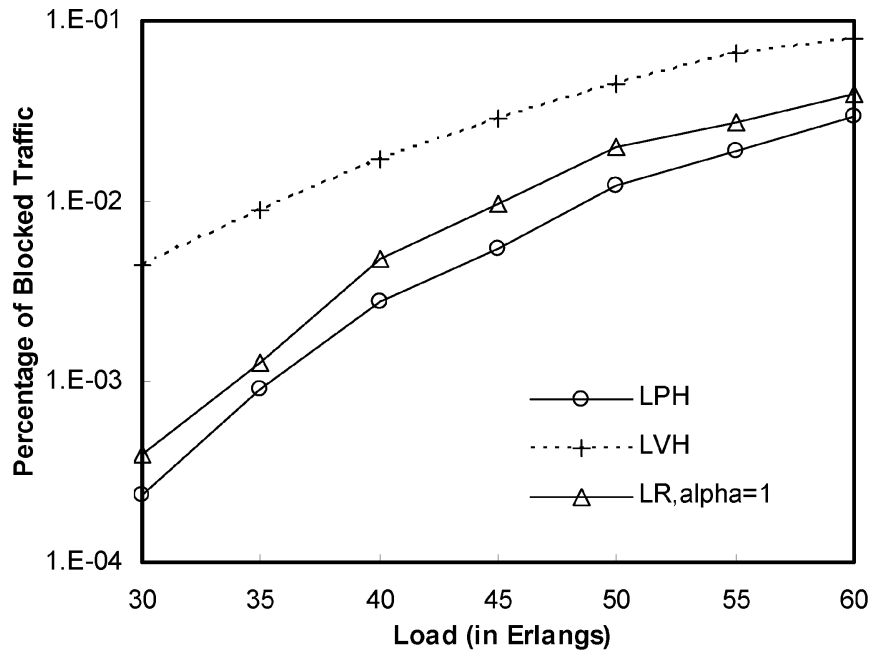

(a)

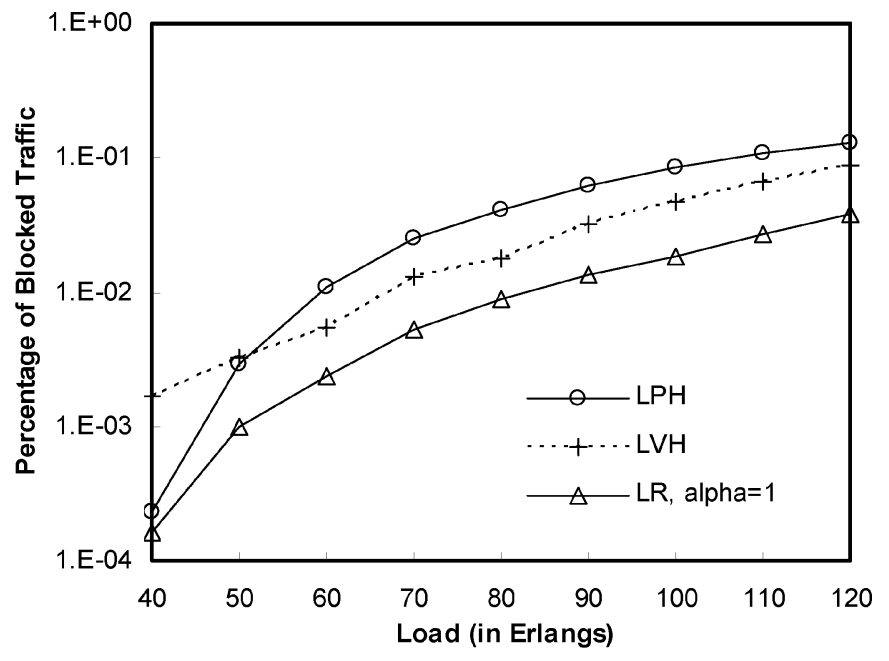

(b)

Fig. 10. Performance of the grooming policies. (a) NSF network, $k=3$. (b) EUPAN network, $k=3$.

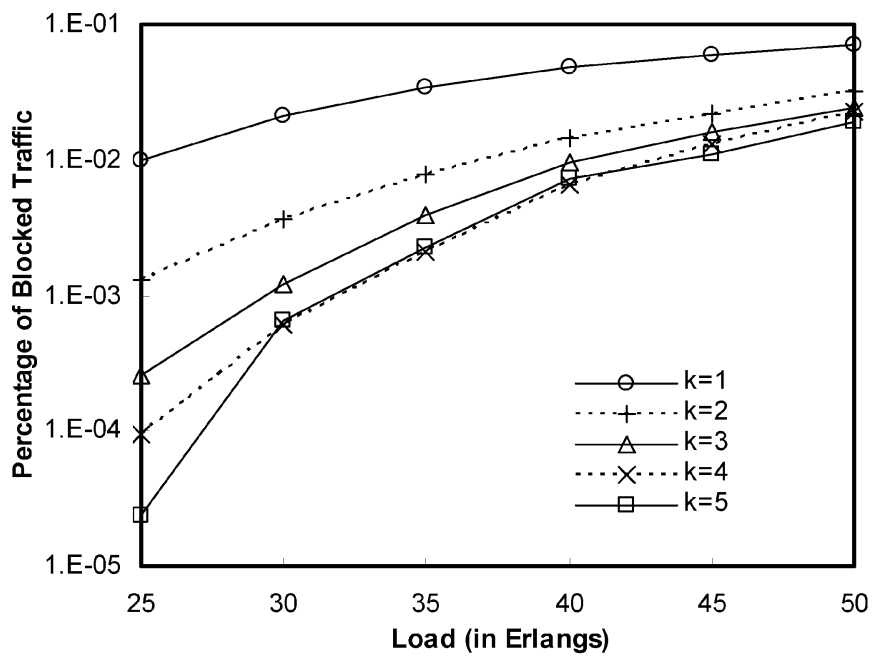

(a)

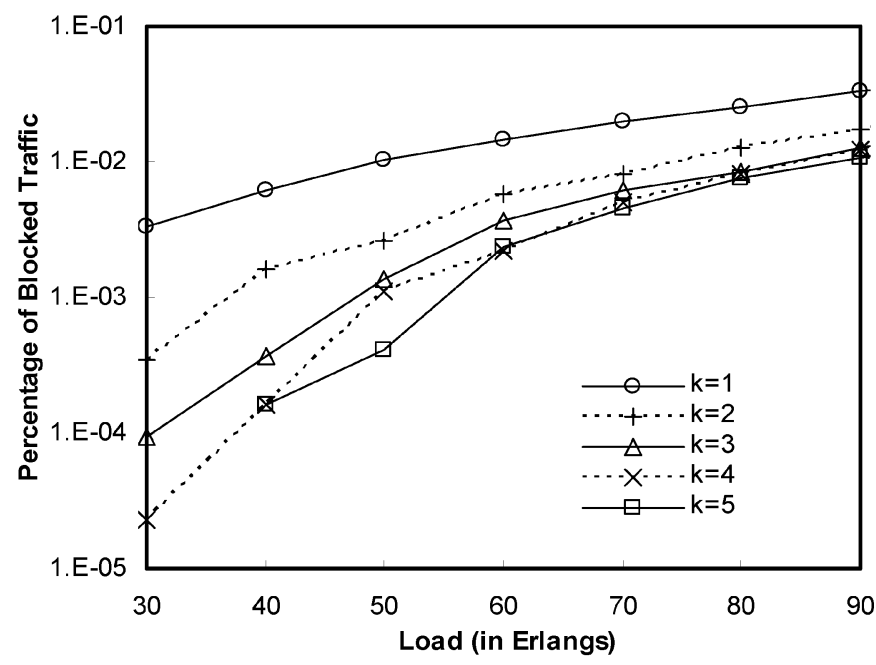

(b)

Fig. 11. Blocking performance of CIGA (without DPP) versus $k$. (a) NSF network. (b) EUPAN network.

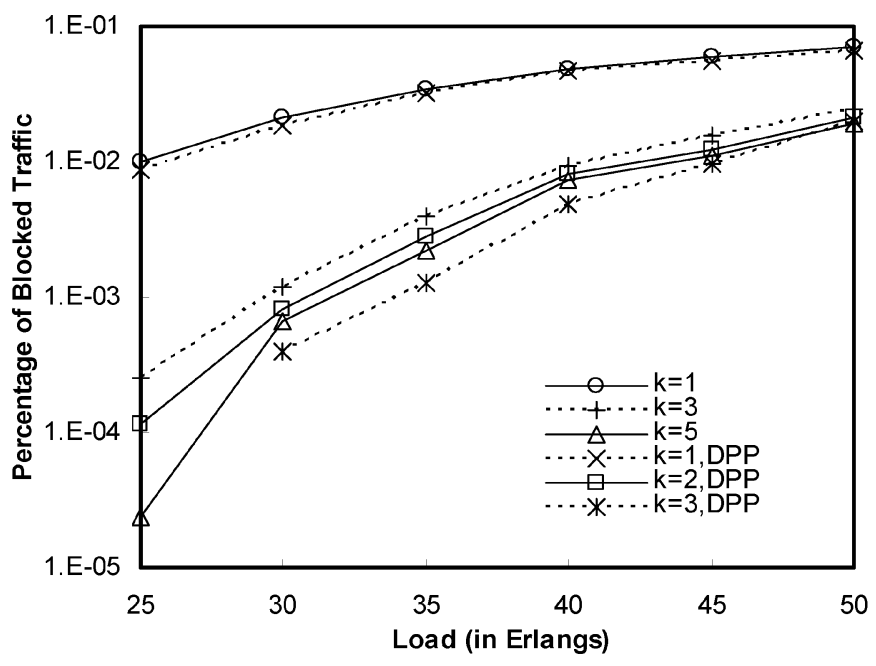

(a)

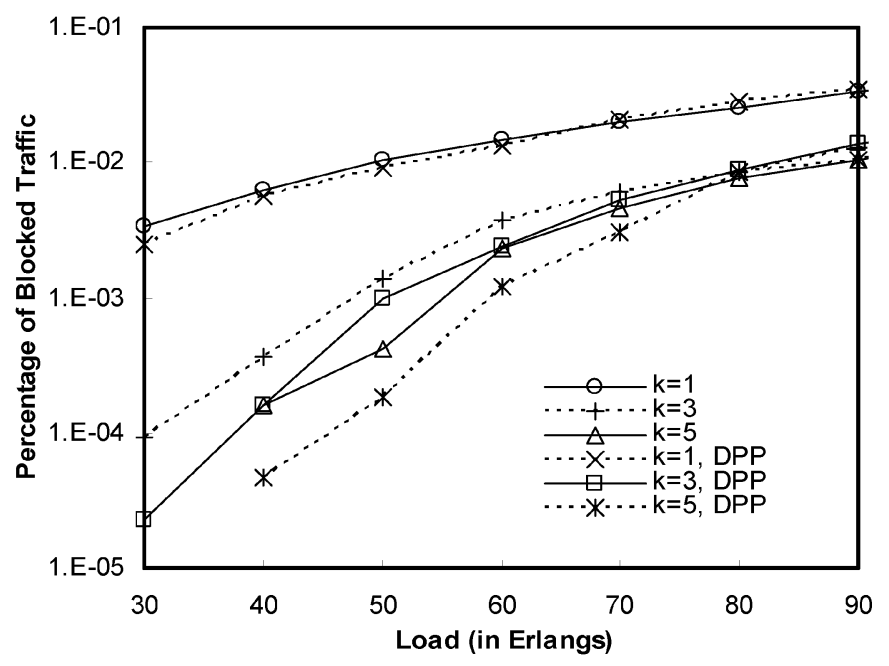

(b)

Fig. 12. Blocking performance of CIGA: DPP versus without DPP. (a) NSF network. (b) EUPAN network. 


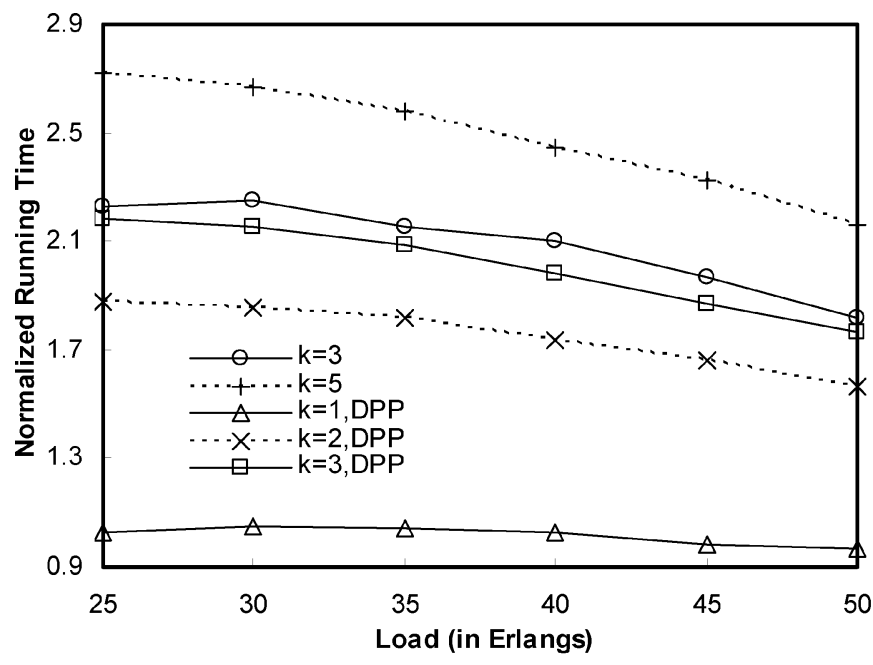

(a)

Fig. 13. Running time of CIGA. (a) NSF network. (b) EUPAN network.

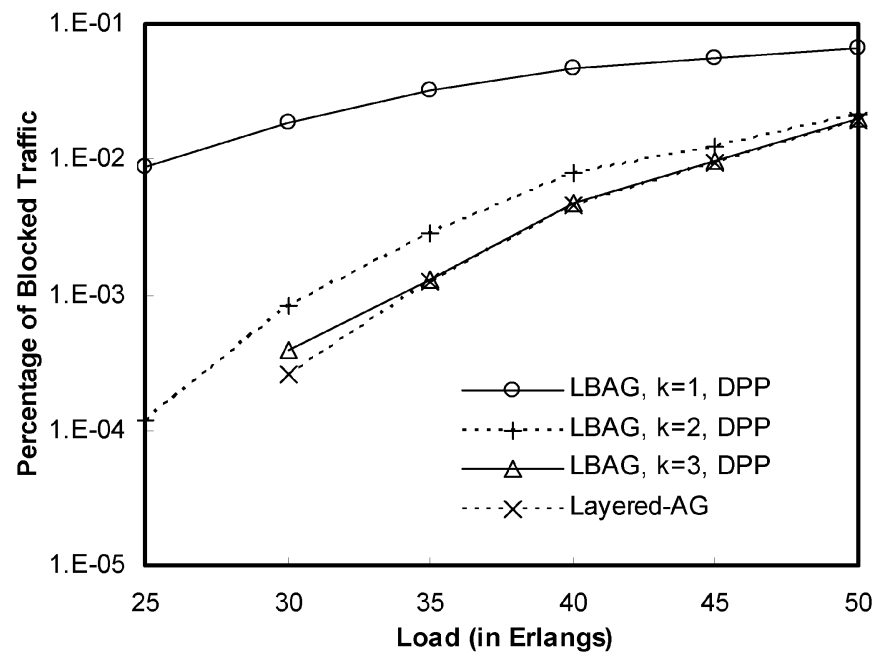

(a)

Fig. 14. Blocking performance of LBAG versus layered-AG. (a) NSF network. (b) EUPAN network. to this assumption. As shown, the running time increases as $k$ increases, regardless of whether DPP is used or not. In addition, even though CIGA_DPP with $k=2$ outperforms CIGA_NO_DPP with $k=3$ in blocking probability in Fig. 12(a), the former still has a shorter running time than the latter in Fig. 13(a). The same results can be found for CIGA_DPP with $k=3$ and CIGA_NO_DPP with $k=5$ in Fig. 13(a). This can be attributed to the ability of DPP to be able to eliminate unnecessary paths at intermediate nodes. The EUPAN network has shown similar results in Fig. 13(b).

\section{Grooming Algorithm Comparison}

In this section, we compare the performance of the proposed LBAG model with the conventional layered auxiliary graph (layered-AG) model [15] in terms of blocking probability and running time. To compare the two models, we compare the performance of the grooming algorithms which are based on the two models, respectively. For the LBAG model, the CIGA algorithm with DPP is used. For the layered-AG model,

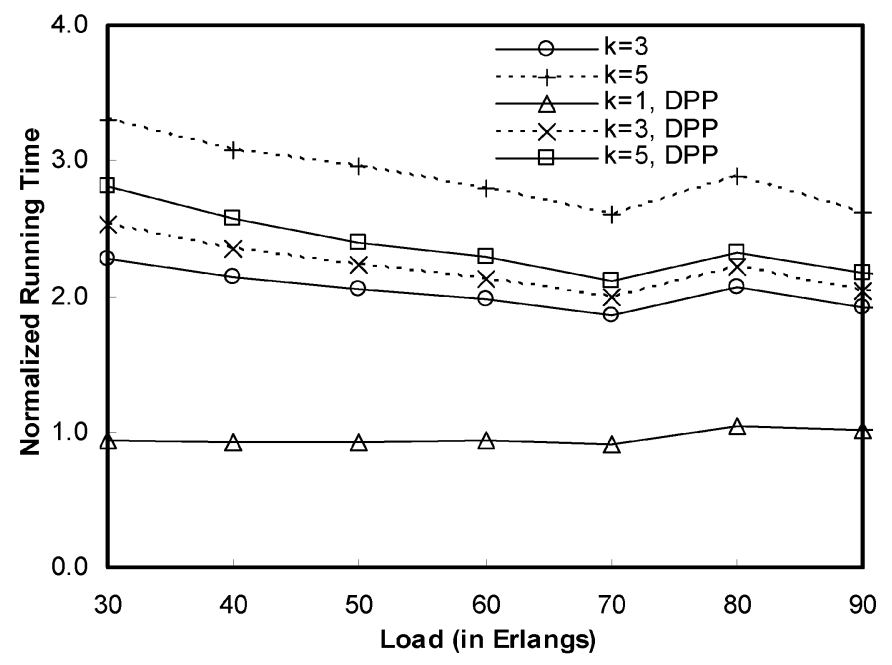

(b)

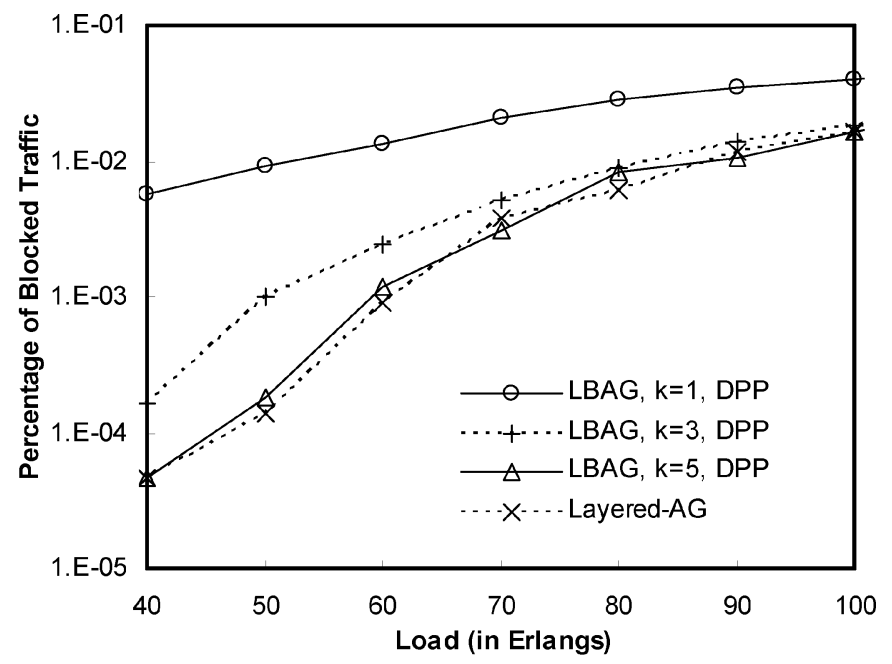

(b) a Dikjstra-based grooming algorithm is used. As stated in the previous sections, the LBAG model generates a largely simplified auxiliary graph than the Layered-AG model. LBAG is expected to have comparable performance with layered-AG in terms of blocking probability. Meanwhile, time complexity analysis shows LBAG has a lower time complexity than layered-AG.

Fig. 14 shows the performance of LBAG and layered-AG in terms of blocking probability. As can be seen, LBAG quickly approaches layered-AG in blocking performance as $k$ increases. In the NSF network, when $k=3$, LBAG almost generate the same blocking probability as layered-AG. In the EUPAN network, which is a relatively denser network than the NSF network, LBAG achieves comparable blocking probability with layered-AG when $k=5$.

While both models can achieve comparable performance in terms of blocking probability, the LBAG model excels the layered-AG model in grooming algorithm running time. As shown in Fig. 15, the running time of layered-AG is several times larger 


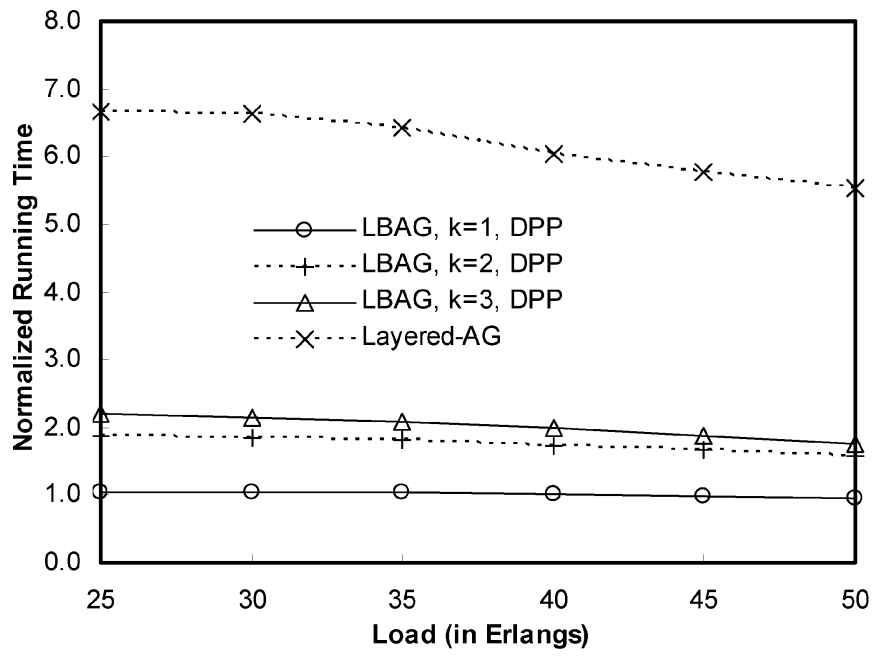

(a)

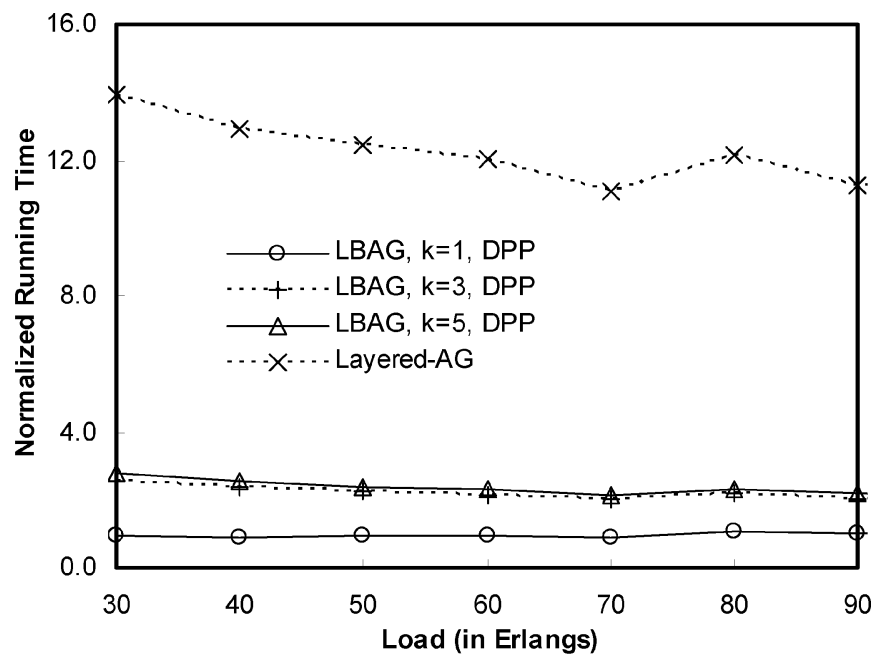

(b)

Fig. 15. Running time of LBAG versus layered-AG. (a) NSF network. (b) EUPAN network.

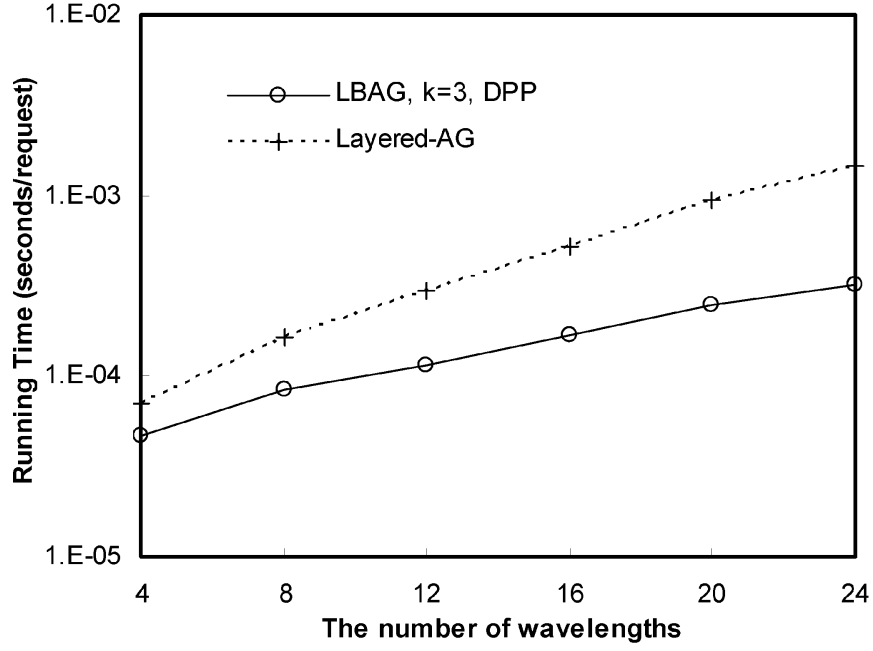

(a)

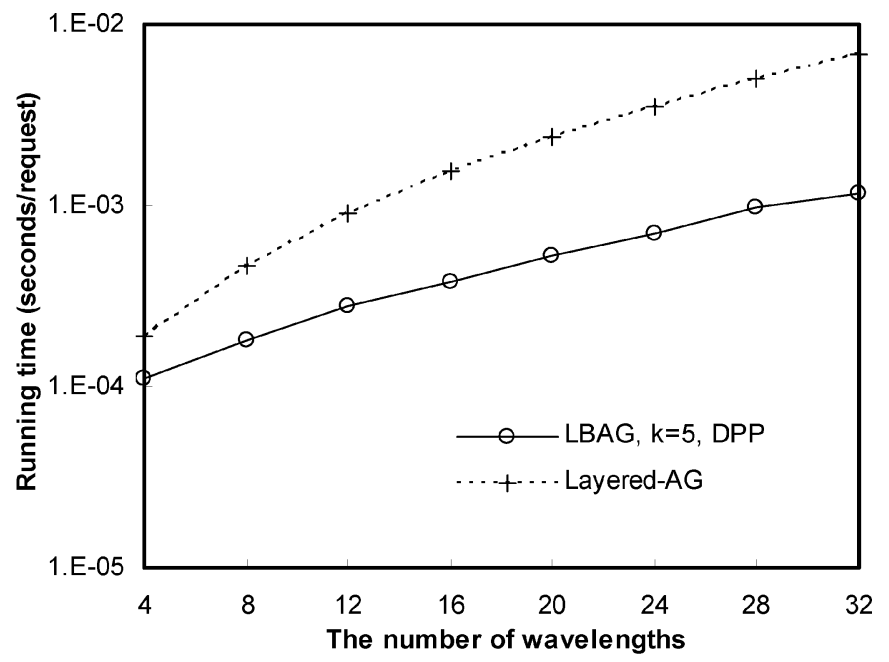

(b)

Fig. 16. Running time of CIGA versus layered-AG approach. (a) NSF network. (b) EUPAN network.

than that of LBAG, for both the NSF network and the EUPAN network. For LBAG, as $k$ increases, the running time also increases. However, the running time increases very slowly with $k$. The running time of LBAG with $k=3$ is just slightly larger than that of LBAG with $k=2$ in the NSF network. The running time of LBAG with $k=5$ is almost equal to that of LBAG with $k=3$ in the EUPAN network. This indicates that the running time of LBAG would not increase unboundedly as $k$ continues to increase. In fact, the running time tends to stop increasing much when $k$ is only a small number such as 3 (for both the NSF network and the EUPAN network).

Fig. 16 illustrates the effect of the number of wavelengths $(W)$ on the running times of the grooming algorithms (grooming auxiliary graph models). As can be seen, both the running times of $\mathrm{LBAG}$ and layered-AG increase as $W$ increases. However, the running time of LBAG increases at a slower pace with $W$ than that of layered-AG. The gap between their running times becomes larger as $W$ increases. As the future WDM optical networks may use tens of wavelengths, the advantage of LBAG over layered-AG in terms of running time will become more promising.

\section{CONCLUSION}

In this paper, we introduced the LBAG model for dynamic traffic grooming problem subject to the GWC constraint. This auxiliary graph has a much smaller number of nodes compared with the conventional auxiliary graph used in the literature. The current advancement of networking technology tends to support traffic engineering (TE) and quality-of-service (QoS) in the network with constrained routing. However, the time complexity of most constrained routing algorithms depends heavily on the size of the network graph. From this perspective, the LBAG model is a promising model to be used in optical networks to support constrained routing. Based on the LBAG model, we proposed the SAG-LB method to address the GWC constraints. With the LBAG model and the SAG-LB method, we proposed the constrained integrated grooming algorithm (CIGA). While 
the CIGA grooming algorithm is constraint-oriented as to solve the GWC constraint, it can be extended to address other constraints specified in TE or QoS.

The proposed CIGA algorithm includes the CGSP algorithm which is used to calculate the shortest available path in LBAG. It incorporates the $k$-shortest path and DPP concepts into the path relaxation process. The worst case time complexity analysis shows that our algorithm has a comparable and in some cases less time complexity compared with the algorithm based on the conventional layered-AG. Our simulation results show that the CIGA algorithm based on the LBAG model can achieve a comparable blocking performance with the grooming algorithm based on the layered-AG model but uses significantly less amount of running time. Although the worst case time complexity analysis shows that both LBAG and layered-AG have time complexities on the order of $W^{2}$, our simulation results show the average running time of LBAG increases slower with $W$ than that of layered-AG. This makes the LBAG model more attractive than layered-AG because a future WDM optical network may support tens of wavelengths. In addition, our simulation results also show that the DPP technique helps in reducing the path search space of the CIGA grooming algorithm. Generally, CIGA with DPP yields a better blocking performance than CIGA without DPP when the parameter $k$ (the number of shortest paths discovered in the relaxation process of CGSP) is the same. Moreover, CIGA with DPP usually has a smaller running time than CIGA without DPP because of the reduced path search space.

We also proposed the least resource path first (LR) grooming policy which integrates the wavelength and transceiver metrics in the edge weight function. The relative weight of wavelength and transceiver is represented using the parameter $\alpha$. Our simulation results show that LR performs consistently well in different scenarios. On the other hand, LPH and LVH, the two existing grooming policies from the literature, can only perform well in wavelength-stringent and transceiver-stringent cases, respectively.

\section{APPENDIX}

Next, we provide the proofs of the theorems presented in Section IV-B. We also prove several lemmas to be used in the proofs of the theorems.

Lemma 1: For a node $v, \lambda^{\prime} \in W_{c}(\{\lambda\}, v)$ if and only if $\lambda \in W_{r}\left(\left\{\lambda^{\prime}\right\}, v\right)$.

Proof: By the definition of $W_{c}$ in (1), $\lambda^{\prime} \in W_{c}(\{\lambda\}, v)$ if and only if $f_{\mathrm{wc}}\left(\lambda, \lambda^{\prime}, v\right)=1$. By the definition of $W_{r}$ in (2), $f_{\mathrm{wc}}\left(\lambda, \lambda^{\prime}, v\right)=1$ if and only if $\lambda \in W_{r}\left(\left\{\lambda^{\prime}\right\}, v\right)$. Hence, the lemma is proved.

Lemma 2: For a path $p\left(v_{0}, v_{1}, \ldots, v_{k}\right)$, if $W_{f}\left(p, v_{k}\right) \neq \varnothing$, then $W_{f}\left(p, v_{i}\right) \neq \varnothing$ for $0 \leq i \leq k$.

Proof: Assume $\exists l, 0 \leq l \leq k-1, W_{f}\left(p, v_{l}\right)=\varnothing$. By the definition of $W_{c}, W_{c}(\varnothing, u)=\varnothing$ for any node $u$. Therefore, $W_{f}\left(p, v_{i}\right)=\varnothing \forall i \geq l$. This is a contradiction to the given condition that $W_{f}\left(p, v_{k}\right) \neq \varnothing$.

Thus, $W_{f}\left(p, v_{i}\right)$ must not be $\varnothing$ for all $0 \leq i \leq k$.
Lemma 3: For a path $p\left(v_{0}, v_{1}, \ldots, v_{k}\right)$, if $W_{f}\left(p, v_{k}\right) \neq \varnothing$, then $\forall \lambda^{\prime} \in W_{f}\left(p, v_{i+1}\right), \exists \lambda \in W_{f}\left(p, v_{i}\right)$ such that $\lambda \in W_{r}\left(\left\{\lambda^{\prime}\right\}, v_{i}\right)$, where $1 \leq i \leq k-1$.

Proof: Assume $\forall \lambda \in \bar{W}_{f}\left(p, v_{i}\right), \lambda \notin W_{r}\left(\left\{\lambda^{\prime}\right\}, v_{i}\right)$. By Lemma $1, \lambda \notin W_{r}\left(\left\{\lambda^{\prime}\right\}, v_{i}\right)$ implies $\lambda^{\prime} \notin W_{c}\left(\{\lambda\}, v_{i}\right)$. Thus, from the assumption, we can have $\lambda^{\prime} \notin W_{c}\left(W_{f}\left(p, v_{i}\right), v_{i}\right)$. Because $W_{f}\left(p, v_{i+1}\right)=W_{c}\left(W_{f}\left(p, v_{i}\right), v_{i}\right) \cap W_{a}\left(v_{i}, v_{i+1}\right)$ by definition in (6), $W_{f}\left(p, v_{i+1}\right) \subseteq W_{c}\left(W_{f}\left(p, v_{i}\right), v_{i}\right)$. Thus, $\lambda^{\prime} \notin$ $W_{c}\left(W_{f}\left(p, v_{i}\right), v_{i}\right)$ implies $\lambda^{\prime} \notin W_{f}\left(p, v_{i+1}\right)$. This is a contradiction to the given condition that $\lambda^{\prime} \in W_{f}\left(p, v_{i+1}\right)$. Therefore, $\exists \lambda \in W_{f}\left(p, v_{i}\right), \lambda \in W_{r}\left(\left\{\lambda^{\prime}\right\}, v_{i}\right)$. By Lemma 2, the condition $W_{f}\left(p, v_{k}\right) \neq \varnothing$ ensures that there are available wavelengths on all the edges, instead of being a null wavelength for some $\lambda$ and $\lambda^{\prime}$.

Theorem 1: For a path $p\left(v_{0}, v_{1}, \ldots, v_{k}\right)$, if $W_{f}\left(p, v_{k}\right) \neq \varnothing$, then (13) gives a feasible wavelength assignment satisfying the GWC constraint

$$
\begin{aligned}
& \lambda_{i} \\
& =\left\{\begin{array}{ll}
\forall \lambda \in W_{f}\left(p, v_{k}\right), & \text { if } i=k \\
\forall \lambda \in\left(W_{r}\left(\left\{\lambda_{i+1}\right\}, v_{i}\right) \cap W_{f}\left(p, v_{i}\right)\right), & \text { if } 1 \leq i \leq k-1
\end{array} .\right.
\end{aligned}
$$

Proof: To prove that the wavelength assignment scheme satisfies the GWC constraint, we must show the two conditions specified in (3) and (4) are met. When $i=k, W_{f}\left(p, v_{k}\right) \neq \varnothing$ is a given condition. Thus, $\exists \lambda_{k} \in W_{f}\left(p, v_{k}\right)$. When $1 \leq i \leq$ $k-1$, by Lemma 3 , we know that $W_{r}\left(\left\{\lambda_{i+1}\right\}, v_{i}\right) \cap W_{f}\left(p, v_{i}\right) \neq$ $\varnothing$. This implies that $\exists \lambda_{i} \in W_{r}\left(\left\{\lambda_{i+1}\right\}, v_{i}\right) \cap W_{f}\left(p, v_{i}\right) \subseteq$ $W_{f}\left(p, v_{i}\right)$.

Thus, for $1 \leq i \leq k$

$$
\begin{aligned}
\exists \lambda_{i} \in W_{f}\left(p, v_{i}\right)=\left(W_{c}\left(W_{f}\left(p, v_{i-1}\right), v_{i-1}\right)\right. & \left.\cap W_{a}\left(v_{i-1}, v_{i}\right)\right) \\
& \subseteq W_{a}\left(v_{i-1}, v_{i}\right) .
\end{aligned}
$$

The first condition in (3) is met. To prove that the second condition in (4) is met, it is enough to show that $\lambda_{i-1} \in$ $W_{r}\left(\left\{\lambda_{i}\right\}, v_{i-1}\right)$ for $2 \leq i \leq k$ by Lemma 1 . By definition in (13), we have $\lambda_{i-1} \in W_{r}\left(\left\{\lambda_{i}\right\}, v_{i-1}\right) \cap W_{f}\left(p, v_{i-1}\right) \subseteq$ $W_{r}\left(\left\{\lambda_{i}\right\}, v_{i-1}\right)$ for $2 \leq i \leq k$. Thus, the theorem is proved.

Theorem 2: For a path $p\left(v_{0}, v_{1}, \ldots, v_{k}\right)$, the GWC constraint is satisfied if and only if $W_{f}\left(p, v_{k}\right) \neq \varnothing$.

Proof: First, we prove that $W_{f}\left(p, v_{k}\right) \neq \varnothing$ if the GWC constraint is satisfied by mathematical induction. $W_{f}\left(p, v_{0}\right)=W \neq \varnothing . W_{c}\left(W_{f}\left(p, v_{0}\right), v_{0}\right)=W_{c}\left(W, v_{0}\right)=W$. Thus, $W_{f}\left(p, v_{1}\right)=W \cap W_{a}\left(v_{0}, v_{1}\right)=W_{a}\left(v_{0}, v_{1}\right)$ and $\lambda_{1} \in W_{a}\left(v_{0}, v_{1}\right)=W_{f}\left(p, v_{1}\right)$. Assume that $W_{f}\left(p, v_{i-1}\right) \neq \varnothing$ and $\lambda_{i-1} \in W_{f}\left(p, v_{i-1}\right)$ for some $1 \leq i \leq k$, we must show that $W_{f}\left(p, v_{i}\right) \neq \varnothing$ and $\lambda_{i} \in W_{f}\left(p, v_{i}\right)$. According to (3) and (4), $\exists \lambda_{i-1} \in W_{a}\left(v_{i-2}, v_{i-1}\right)$ and $\exists \lambda_{i} \in W_{a}\left(v_{i-1}, v_{i}\right)$ such that $\lambda_{i} \in W_{c}\left(\left\{\lambda_{i-1}\right\}, v_{i-1}\right)$. Thus, $W_{c}\left(\left\{\lambda_{i-1}\right\}, v_{i-1}\right) \cap W_{a}\left(v_{i-1}, v_{i}\right) \neq \varnothing$.

Because $\lambda_{i-1} \in W_{f}\left(p, v_{i-1}\right), W_{c}\left(\left\{\lambda_{i-1}\right\}, v_{i-1}\right) \subseteq$ $W_{c}\left(W_{f}\left(p, v_{i-1}\right), v_{i-1}\right)$ by the definition of $W_{c}$.

Thus, $W_{f}\left(p, v_{i}\right)=W_{c}\left(W_{f}\left(p, v_{i-1}\right), v_{i-1}\right) \cap$ $W_{a}\left(v_{i-1}, v_{i}\right) \neq \varnothing$. In addition

$$
\begin{aligned}
\lambda_{i} \in & \left(W_{c}\left(\left\{\lambda_{i-1}\right\}, v_{i-1}\right) \cap W_{a}\left(v_{i-1}, v_{i}\right)\right) \\
& \subseteq\left(W_{c}\left(W_{f}\left(p, v_{i-1}\right), v_{i-1}\right) \cap W_{a}\left(v_{i-1}, v_{i}\right)\right)=W_{f}\left(p, v_{i}\right) .
\end{aligned}
$$

This completes the proof that $W_{f}\left(p, v_{i}\right) \neq \varnothing$ for $0 \leq i \leq k$. 
From Theorem 1, we know that if $W_{f}\left(p, v_{k}\right) \neq \varnothing$, a feasible wavelength assignment satisfying the GWC constraint exists. Thus, the theorem is proved.

\section{REFERENCES}

[1] K. Zhu and B. Mukherjee, "Traffic grooming in an optical WDM mesh network," IEEE J. Sel. Areas Commun., vol. 20, no. 1, pp. 122-133, Jan. 2002.

[2] O. Gerstel, P. Lin, and G. Sasaki, "Combined WDM and SONET network design," in Proc. IEEE INFOCOM, vol. 2, New York, Mar. 1999, pp. 734-743.

[3] A. L. Chiu and E. Modiano, "Traffic grooming algorithms for reducing electronic multiplexing costs in WDM ring networks," IEEE/OSA J. Lightw. Technol., vol. 18, no. 1, pp. 2-12, Jan. 2000.

[4] J. Wang, V. R. Vemuri, W. Cho, and B. Mukherjee, "Improved approaches for cost-effective traffic grooming in WDM ring networks: ILP formulations and single-hop and multihop connections," IEEE/OSA J. Lightw. Technol., vol. 19, no. 11, pp. 1645-1653, Nov. 2001.

[5] S. Zhang and B. Ramamurthy, "Dynamic traffic grooming algorithms for reconfigurable SONET over WDM networks," IEEE J. Sel. Areas Commun., vol. 21, no. 7, pp. 1165-1172, Sep. 2003.

[6] B. Ramamurthy and B. Mukherjee, "Wavelength conversion in WDM networking," IEEE J. Sel. Areas Commun., vol. 16, no. 7, pp. 1061-1073, Sep. 1998.

[7] J. Yates, J. Lacey, D. Everitt, and M. Summerfield, "Limited-range wavelength translation in all-optical networks," in Proc. INFOCOM, Mar. 1996, pp. 954-961.

[8] S. Subramaniam, M. Azizoglu, and A. K. Somani, "All-optical networks with sparse wavelength conversion," IEEE/ACM Trans. Netw., vol. 4, no. 4, pp. 544-557, Aug. 1996.

[9] S. Dixit, IP Over WDM: Building the Next-Generation Optical Internet. New York: Wiley, 2003.

[10] E. Mannie. (2003) Generalized multiprotocol label switching (GMPLS) architecture. Internet draft. [Online]. Available: draft-ietf-ccamp-gmplsarchitecture-04.txt

[11] E. Rosen, A. Viswanathan, and R. Callon, "Multiprotocol label switching architecture," IETF, RFC 3031, Jan. 2001.

[12] K. Zhu and B. Mukherjee, "On-line approaches for provisioning connections of different bandwidth granularities in WDM mesh networks," in Proc. OFC, 2002, pp. 549-551.

[13] C. Xin, Y. Ye, S. Dixit, and C. Qiao, "An integrated lightpath provisioning approach in mesh optical networks," in Proc. OFC, 2002, pp. $547-549$

[14] H. Zhu, H. Zang, K. Zhu, and B. Mukherjee, "Dynamic traffic grooming in WDM mesh networks using a novel graph model," Proc. IEEE GLOBECOM, pp. 2681-2685, Nov. 2002.

[15] —_, "A novel, generic graph model for traffic grooming in heterogeneous WDM mesh networks," IEEE/ACM Trans. Netw., vol. 11, no. 2, pp. 285-299, Apr. 2003.

[16] C. Chen and S. Banerjee, "A new model for optimal routing and wavelength assignment in wavelength division multiplexed optical networks," in Proc. INFOCOM, vol. 2, Mar. 1996, pp. 164-171.

[17] E. I. Chong, S. Maddila, and S. Morley, "On finding single-source single-destination $k$ shortest paths," J. Comput. Inf., pp. 40-47, 1995.

[18] P. V. Meighem, H. D. Neve, and F. A. Kuipers, "Hop-by-hop quality of service routing," Comput. Netw., vol. 37/3-4, pp. 407-423, Oct. 2001.
[19] A. Lardies, R. Gupta, and R. A. Patterson, "Traffic grooming in a multilayer network," Opt. Netw. Mag., vol. 2, no. 3, pp. 91-99, May/Jun. 2001.

[20] H. Zang, J. P. Jue, and B. Mukherjee, "A review of routing and wavelength assignment approaches for wavelength-routed optical WDM networks," Opt. Netw. Mag., vol. 1, no. 1, pp. 47-60, Jan. 2000.

[21] T. H. Cormen, C. E. Leiserson, and R. L. Rivest, Introduction to Algorithms. Cambridge, MA: MIT Press, 1990.

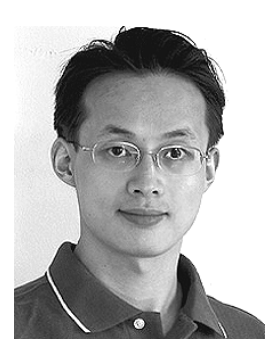

Wang Yao (S'02) received the B.E. and M.E. degrees from the Beijing University of Aeronautics and Astronautics, Beijing, China, in 1997 and 2000, respectively. $\mathrm{He}$ is currently working towards the Ph.D. degree in the Department of Computer Science and Engineering, University of Nebraska, Lincoln (UNL).

Before he joined UNL, he was with Huawei Beijing Research Center, Huawei Technologies, for one year as a Senior Software Engineer. His research interests include networking and distributed computing, optical communication networks, and wireless networks.

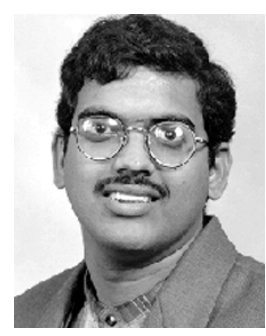

Byrav Ramamurthy (S'97-M'98) received the B.Tech. degree in computer science and engineering from the Indian Institute of Technology, Madras, in 1993, and the M.S. and Ph.D. degrees in computer science from the University of California (UC), Davis, in 1995 and 1998, respectively.

Since August 2003, he has been an Associate Professor in the Department of Computer Science and Engineering, University of Nebraska, Lincoln (UNL). He is the Co-Director of the UNL Academic Program Priority Initiative in the areas of Simulation and Computing Engineering (SCE) and Information Technology and Telecommunications (ITT). He is the founding Co-Director of the Advanced Networking and Distributed Experimental Systems (ANDES) Laboratory, UNL. He is author of Design of Optical WDM Networks-LAN, MAN and WAN Architectures (Norwell, MA: Kluwer, 2000). His research areas include optical networks, wireless networks, network security, distributed computing, and telecommunications.

Dr. Ramamurthy was a recipient of the Indian National Talent Search Scholarship and was a Fellow of the Professors for the Future Program, UC Davis. He is a recipient of the UNL Research Council Grant-in-Aid Award for 1999 , the College of Engineering and Technology Faculty Research Award for 2000, and the UNL CSE Department Students Choice Award for the Best Graduate Professor for 2002 to 2003. He was the Feature Editor on Theses for the Optical Network Magazine. He served as a Guest Co-Editor for the IEEE Network Magazine (Special Issue on Optical Communication Networks). He served as a Member of the Technical Program Committees for the IEEE INFOCOM, the IEEE GLOBECOM, Opticomm, ICC, and the ICCCN Conferences. From 2001 to 2003, he served as the founding Secretary of the IEEE ComSoc Optical Networking Technical Committee (ONTC), for which he is currently the Online Content Chair 